\title{
KAROUBI'S RELATIVE CHERN CHARACTER, THE RIGID SYNTOMIC REGULATOR, AND THE BLOCH-KATO EXPONENTIAL MAP
}

\author{
GEORG TAMME \\ Fakultät für Mathematik, Universität Regensburg, 93040 Regensburg, Germany; \\ email: georg.tamme@mathematik.uni-regensburg.de
}

Received 27 September 2013; accepted 25 July 2014

\begin{abstract}
We construct a variant of Karoubi's relative Chern character for smooth separated schemes over the ring of integers in a $p$-adic field, and prove a comparison with the rigid syntomic regulator. For smooth projective schemes, we further relate the relative Chern character to the étale $p$-adic regulator via the Bloch-Kato exponential map. This reproves a result of Huber and Kings for the spectrum of the ring of integers, and generalizes it to all smooth projective schemes as above.
\end{abstract}

2010 Mathematics Subject Classification: 19F27 (primary); 14F43, 14F30 (secondary)

\section{Introduction}

If $A$ is a Banach algebra, one can view it as an abstract ring and consider its algebraic K-theory, or one can take the topology into account and then consider its topological K-theory. There is a natural map from the former to the latter, and so one can form the homotopy fibre of this map giving the relative K-theory. Karoubi's relative Chern character [Kar83, Kar87, CK88] is a homomorphism

$$
K_{i}^{\mathrm{rel}}(A) \rightarrow H C_{i-1}(A)
$$

mapping relative K-theory to continuous cyclic homology. It was an idea of Karoubi [Kar82, Kar83] that the relative Chern character could be used for the construction of regulators. In accordance with this idea, Hamida [Ham00]

(c) The Author 2014. This is an Open Access article, distributed under the terms of the Creative Commons Attribution licence (http://creativecommons.org/licenses/by/3.0/), which permits unrestricted re-use, distribution, and reproduction in any medium, provided the original work is properly cited. 
established, for $A=\mathbf{C}$ the field of complex numbers, a precise relation between the relative Chern character and the Borel regulator [Bor74]

$$
K_{2 n-1}(\mathbf{C}) \rightarrow \mathbf{C} \text {. }
$$

Karoubi's construction works equally well in the case of ultrametric Banach algebras, and building on previous work by Hamida [Ham06] we proved the $p$-adic analogue of the above result in [Tam12a], giving the precise relation of the relative Chern character with the $p$-adic Borel regulator introduced by Huber and Kings [HK11].

Changing perspective, let $X$ be a smooth variety over C. Again we have the algebraic K-theory of $X$, but we can also consider the topological K-theory of the complex manifold associated to $X$. It is natural to ask for a generalization of the previous results to this situation. Here, the analogues of the cyclic homology groups are the quotients of the de Rham cohomology by the Hodge filtration, and Borel's regulator is replaced by Beilinson's regulator mapping algebraic Ktheory to Deligne-Beilinson cohomology. In this set-up we proved a comparison between the relative Chern character and Beilinson's regulator in [Tam12b].

It is the goal of the present paper to prove the $p$-adic analogue of this result. Let $R$ be a complete discrete valuation ring with field of fractions $K$ of characteristic 0 with perfect residue field $k$ of characteristic $p$, and consider a smooth $R$-scheme $X$. The $p$-adic analogue of Beilinson's regulator is the rigid syntomic regulator, i.e. the Chern character with values in rigid syntomic cohomology, introduced by Gros [Gro94] and developed systematically by Besser [Bes00b]. We introduce topological and hence relative $\mathrm{K}$-theory of $X$, and relative cohomology groups $H_{\text {rel }}^{*}(X, n)$ mapping naturally to the rigid syntomic cohomology groups. These are the target of the relative Chern character in the $p$-adic situation. Our main result is the following.

THEOREM. Let $X$ be a smooth $R$-scheme, and let $i>0$. The diagram

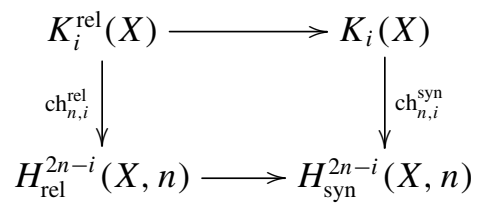

commutes.

If $X$ is proper, the lower horizontal map is in fact an isomorphism, and both groups are given by $H_{\mathrm{dR}}^{2 n-i-1}\left(X_{K} / K\right) / F^{n} H_{\mathrm{dR}}^{2 n-i-1}\left(X_{K} / K\right)$, which, in turn, is naturally isomorphic to the weight $n-1$ part $H C_{i-1}^{(n-1)}\left(X_{K}\right)$ in the $\lambda$-decomposition 
of the cyclic homology of $X_{K} / K$ [Wei97]. Moreover, for projective $X$ and finite $k$, Parshin's conjecture would imply that also the upper horizontal map is rationally an isomorphism.

One of the possible advantages of this approach to the syntomic regulator is that Karoubi's constructions give quite explicit formulae. For instance, in the case when $X=\operatorname{Spec}(R)$, these have been used in the comparison of Karoubi's regulator with the $p$-adic Borel regulator [Tam12a] and in computer calculations by Choo and Snaith [CS11].

Another motivation to study the relative Chern character and its relation to the syntomic regulator goes back to an idea of Besser. In contrast to the Beilinson regulator or Soulé's étale $p$-adic regulator, the rigid syntomic regulator explicitly depends on the choice of the local model $X / R$ of the generic fibre $X_{K} / K$. In computations (e.g. [Bes12, BdJ12]) this leads to integrality assumptions that one would like to remove. Besser proposed the use of Karoubi's relative Chern character in order to obtain a model-independent replacement for the syntomic regulator. In fact, one can define topological and hence relative K-theory of $K$-schemes using the associated rigid space, and the techniques of this paper give a relative Chern character $K_{i}^{\text {rel }}\left(X_{K}\right) \rightarrow H_{\mathrm{dR}}^{2 n-i-1}\left(X_{K} / K\right) / F^{n}$. If $X$ is a smooth proper $R$-scheme, there is a natural map $K_{*}^{\text {rel }}(X) \rightarrow K_{*}^{\text {rel }}\left(X_{K}\right)$, and the relative Chern character for $X$ factors through the relative Chern character of $X_{K}$.

In particular, if one assumes Parshin's conjecture, the relative Chern character would give a description of the syntomic regulator for $X$ smooth projective over $R$ with finite residue field solely in terms of the generic fibre $X_{K}$. In general, a good understanding of topological K-theory is still missing.

From the theorem we also get the following corollary (see 6.16).

COROllary. Assume that $k$ is finite and that $X / R$ is smooth and projective. Then

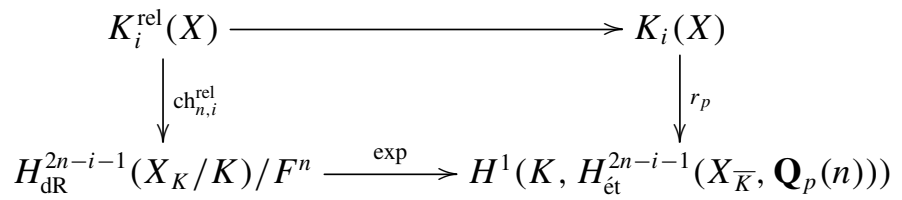

commutes.

Here, $r_{p}$ is the étale $p$-adic regulator and exp is the Bloch-Kato exponential map of the $p$-adic $\operatorname{Gal}(\bar{K} / K)$-representation $H_{\text {ét }}^{2 n-i-1}\left(X_{\bar{K}}, \mathbf{Q}_{p}(n)\right)$. This corollary may be seen as a generalization of the main result of Huber and Kings [HK11, Theorem 1.3.2], which is the case when $X=\operatorname{Spec}(R)$, and amounts to the 
commutativity of

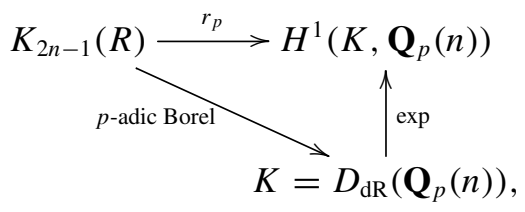

to all smooth projective $R$-schemes (see our Corollary 6.18).

A result related to ours is proven by Chiarellotto et al. in [CCM13]. They provide an alternative construction of the rigid syntomic regulator in terms of higher Chow groups and syntomic cycle classes. A key step in their construction is the compatibility of the de Rham and rigid cycle classes under the specialization map from de Rham cohomology of the generic fibre to rigid cohomology of the special fibre, whereas our result in some sense rests on the compatibility of topological and rigid Chern classes.

For an interpretation of the relative cohomology groups, introduced here, in stable homotopy theory of schemes, we refer the reader to [DM12].

Let us describe the contents of this paper in more detail. Karoubi's original construction of the relative Chern character uses integration of certain $p$-adic differential forms over standard simplices. A reformulation of this construction is given in Section 7. The key ingredient that enables us to compare the relative Chern character and the rigid syntomic regulator is a new description of the former in Section 5 that is similar to the construction of Chern class maps on higher K-groups by Beilinson [Beǐ4], Huber [Hub95], and Besser [Bes00b]. This is made possible by the functorial complexes of Besser [Bes00b] and Chiarellotto, Ciccioni, and Mazzari [CCM13]. Their construction is recalled in Section 4, with some simplifications coming from the systematic use of Große-Klönne's dagger spaces [GK99, GK00]. The necessary background from rigid respectively 'dagger' geometry is collected in Section 2. In Section 3, we recall Karoubi and Villamayor's definition of topological K-theory for ultrametric Banach rings [KV71, Cal85], and extend it to smooth separated $R$-schemes. The necessary comparison between the two constructions of the relative Chern character is proved in Theorem 7.20. The main comparison theorem (Theorem 6.12) then follows rather formally. Applications are given in Section 6.4.

1.1. Notation. For $p \in\{0,1,2, \ldots\}$, we denote by $[p]$ the finite set $\{0, \ldots, p\}$ with its natural order. The category of finite ordered sets with monotone maps is the simplicial category $\Delta$. The unique injective map $[p-1] \rightarrow[p]$ that does not hit $i$ is denoted by $\partial^{i}$. Similarly, $s^{i}:[p+1] \rightarrow[p]$ is the unique surjective map such that $s^{i}(i)=s^{i}(i+1)$. These induce morphisms 
$\partial_{i}, s_{i}$ (respectively, $\partial^{i}, s^{i}$ ) on every (co)simplicial object, called (co)face and (co)degeneracy morphisms, respectively.

For a group object $G$, we define the simplicial objects $E_{\bullet} G$ and $B_{\bullet} G$ by $E_{p} G=G^{\times(p+1)}$ and $B_{p} G=G^{\times p}$, with the usual faces and degeneracies (see, e.g., [HK11, 0.2]).

\section{Preliminaries on rigid geometry}

In the definition of rigid cohomology, one usually works with rigid analytic spaces and their de Rham cohomology. However, as de Rham cohomology is not well behaved for rigid spaces, one has to introduce some overconvergence condition. An elegant approach to do this is to replace rigid spaces by GroßeKlönne's dagger spaces [GK99, GK00]. We recall some basic definitions and facts which will be needed in the rest of the paper.

Let $R$ be a complete discrete valuation ring with field of fractions $K$ of characteristic 0 and residue field $k$ of characteristic $p>0$. Fix an absolute value |. | on $K$.

2.1 (Rings). The $K$-algebra of overconvergent power series in $n$ variables $\underline{x}=$ $\left(x_{1}, \ldots, x_{n}\right)$ is $K\langle\underline{x}\rangle^{\dagger}:=\left\{\sum a_{v} \underline{x}^{v}\left|a_{v} \in K, \exists \rho>1:\right| a_{v} \mid \rho^{|v|} \stackrel{|v| \rightarrow \infty}{\longrightarrow} 0\right\}$. We denote by $R\langle\underline{x}\rangle^{\dagger}$ the sub-R-algebra of overconvergent power series with $R$-coefficients. A $K$ - (respectively, $R$-)dagger algebra is a quotient of some $K\langle\underline{x}\rangle^{\dagger}$ (respectively, $\left.R\langle\underline{x}\rangle^{\dagger}\right)$.

The algebra of overconvergent power series carries the Gauß norm $\left|\sum_{\nu} a_{\nu} \underline{x}^{v}\right|=$ $\sup _{v}\left|a_{v}\right|$. Its completion with respect to this norm is the Tate algebra of convergent power series $K\langle\underline{x}\rangle=\left\{\sum_{v} a_{v} x^{v}|| a_{v} \mid \stackrel{|v| \rightarrow \infty}{\longrightarrow} 0\right\}$. Similarly, the completion of $R\langle x\rangle^{\dagger}$ is $R\langle x\rangle$. Quotients of these are called $K$ - (respectively, $R$-) affinoid algebras. These are Banach algebras. Up to equivalence, the quotient norm on a dagger or affinoid algebra does not depend on the chosen representation as a quotient of an algebra of overconvergent (respectively, convergent) power series.

To any $R$-algebra $A$ one can associate its weak completion $A^{\dagger}$ (see [MW68, Definition 1.1]). If $A$ is an $R$-algebra of finite type and $A \cong R[\underline{x}] / I$ is a presentation, then there is an induced isomorphism $A^{\dagger} \cong R\langle\underline{x}\rangle^{\dagger} / I R\langle\underline{x}\rangle^{\dagger}$. In particular, $A^{\dagger}$ is an $R$-dagger algebra. Similarly, there is a weak completion for normed $K$-algebras.

The categories of $R$-dagger (respectively, $K$-dagger) and affinoid algebras admit tensor products. For example, if $A$ and $B$ are $R$-dagger algebras, their tensor product is $A \otimes_{R}^{\dagger} B:=\left(A \otimes_{R} B\right)^{\dagger}$. Given presentations $A \cong R\langle\underline{x}\rangle^{\dagger} / I$ and $B \cong R\langle\underline{y}\rangle^{\dagger} / J$, there is a natural isomorphism $A \otimes_{R}^{\dagger} B \cong R\langle\underline{x}, \underline{y}\rangle^{\dagger} /(I+J)$. 
2.2 (Spaces). We only sketch the main points here, referring the reader to [GK99, GK00] for details. Similarly as one defines rigid analytic spaces that are locally isomorphic to max-spectra of $K$-affinoid algebras with a certain Grothendieck topology, dagger spaces are defined by taking the max-spectra $\operatorname{Sp}(A)$ of $K$-dagger algebras $A$ as building blocks [GK00, 2.12]. For any dagger space $X$ one has an associated rigid space $X^{\text {rig }}$ ('completion of the structure sheaf') and a natural map of G-ringed spaces $X^{\text {rig }} \stackrel{u}{\rightarrow} X$, which is an isomorphism on the underlying G-topological spaces [GK00, 2.19].

There exists a dagger analytification functor $X \mapsto X^{\dagger}$ from the category of $K$-schemes of finite type to the category of $K$-dagger spaces. There is a natural morphism of locally G-ringed spaces $X^{\dagger} \stackrel{\iota}{\rightarrow} X$ which is final for morphisms from dagger spaces to $X$ [GK00, 3.3].

We also need the notion of weak formal schemes ([GK99, Ch. 3] and originally [Mer72]). Let $A$ be an $R$-dagger algebra, and let $\bar{A}=A \otimes_{R} k$. Then $D(\bar{f}) \mapsto$ $A\langle 1 / f\rangle^{\dagger}$, where $f \in A$ lifts $\bar{f} \in \bar{A}$, defines a sheaf of local rings on the topological space $\operatorname{Spec}(\bar{A})$. The corresponding locally ringed space is the weak formal $R$-scheme $\operatorname{Spwf}(A)$. A general weak formal $R$-scheme is a locally ringed space that is locally isomorphic to some $\operatorname{Spwf}(A)$.

Sending $\operatorname{Spwf}(A)$ for an $R$-dagger algebra $A$ to $\operatorname{Sp}\left(A \otimes_{R} K\right)$ induces the generic fibre functor $\mathscr{X} \mapsto \mathscr{X}_{K}$ from weak formal $R$-schemes to $K$-dagger spaces, and there is a natural specialization map sp: $\mathscr{X}_{K} \rightarrow \mathscr{X}$.

Taking the weak completion of finitely generated $R$-algebras induces the functor $X \mapsto \widehat{X}$ from $R$-schemes of finite type to weak formal $R$-schemes. There is a natural morphism of dagger spaces from the generic fibre of the weak completion of $X, \widehat{X}_{K}$, to the dagger analytification $X_{K}^{\dagger}$ of the generic fibre $X_{K}$ of $X$. This is an open immersion if $X$ is separated and an isomorphism if $X$ is proper over $R$ (see [Ber96, Proposition 0.3.5]). For example, if $X=\mathbb{A}_{R}^{1}$, then $\widehat{X}_{K}=\operatorname{Sp}\left(K\langle x\rangle^{\dagger}\right)$ is the closed ball of radius 1 in $\left(\mathbb{A}_{K}^{1}\right)^{\dagger}$.

\section{Preliminaries on K-theory}

3.1. Let $A$ be an ultrametric Banach ring with norm $|$.$| , for example, an affinoid$ algebra with a fixed norm. In [KV71], Karoubi and Villamayor introduce Kgroups of $A$ that we will denote by $K_{\text {top }}^{-i}(A), i \geqslant 1$, which were further studied by Calvo [Cal85]. A convenient way to define them is the following. Set

$$
A\left\langle x_{0}, \ldots, x_{n}\right\rangle:=\left\{\sum a_{\nu} \underline{x}^{v}\left|a_{\nu} \in A,\right| a_{\nu} \mid \stackrel{|v| \rightarrow \infty}{\longrightarrow} 0\right\}
$$

and $A\left\langle\Delta^{n}\right\rangle:=A\left\langle x_{0}, \ldots, x_{n}\right\rangle /\left(\sum_{i} x_{i}-1\right)$. Then $[n] \mapsto A\left\langle\Delta^{n}\right\rangle$ becomes a simplicial ring in a natural way, and hence $B \cdot \operatorname{GL}\left(A\left\langle\Delta^{\bullet}\right\rangle\right)$ is a bisimplicial set 
(see 1.1 ). For any bisimplicial set $S_{. .}$, we denote by $\pi_{*}\left(S_{\bullet \bullet}\right)$ the homotopy groups of the underlying diagonal simplicial set. We define

$$
K_{\text {top }}^{-i}(A):=\pi_{i}\left(B . \operatorname{GL}\left(A\left\langle\Delta^{\bullet}\right\rangle\right)\right), \quad i \geqslant 1 .
$$

That this definition coincides with the original one in [KV71, Cal85] follows from an argument of Anderson [Tam10, Proposition 7.3].

For our purposes it is important to know that one can compute the K-theory of affinoid algebras using dagger algebras. More precisely, let $R$ be a complete discrete valuation ring with field of fractions $K$ of characteristic 0 and residue field $k$ of characteristic $p>0$, as before. We define the simplicial ring $R\left\langle\Delta^{\bullet}\right\rangle^{\dagger}$ by $R\left\langle\Delta^{n}\right\rangle^{\dagger}=R\left\langle x_{0}, \ldots, x_{n}\right\rangle^{\dagger} /\left(\sum_{i} x_{i}-1\right)$, with the obvious structure maps. For any $R$-dagger algebra $A$, we set $A\left\langle\Delta^{\bullet}\right\rangle^{\dagger}=A \otimes_{R}^{\dagger} R\left\langle\Delta^{\bullet}\right\rangle^{\dagger}$ (see 2.1), and define topological K-groups by

$$
K_{\text {top }}^{-i}(A):=\pi_{i}\left(B \cdot G L\left(A\left\langle\Delta^{\bullet}\right\rangle^{\dagger}\right)\right), \quad i \geqslant 1 .
$$

Using Calvo's techniques, we have shown in [Tam10, Proposition 7.5] that these agree with the Karoubi-Villamayor K-groups of the completion $\widehat{A}$ of $A$ :

$$
K_{\text {top }}^{-i}(A) \cong K_{\text {top }}^{-i}(\widehat{A}), \quad i \geqslant 1 .
$$

Now, let $X=\operatorname{Spec}(A)$ be an affine scheme of finite type over $R$.

Definition 1. We define the topological K-groups of $X$ to be the topological K-groups of the (weak) completion of $A$ :

$$
K_{\text {top }}^{-i}(X):=K_{\text {top }}^{-i}\left(A^{\dagger}\right)=K_{\text {top }}^{-i}(\widehat{A}), \quad i \geqslant 1 .
$$

REMARKS 3.2. (i) Note the similarity with topological complex K-theory: if $X$ is a smooth separated scheme of finite type over $\mathbf{C}$, and $A_{\bullet}$ denotes the simplicial ring of smooth functions $X(\mathbf{C}) \times \Delta^{\bullet} \rightarrow \mathbf{C}$, then $\pi_{i}\left(B_{\bullet} \operatorname{GL}\left(A_{\bullet}\right)\right) \cong$ $K_{\text {top }}^{-i}(X(\mathbf{C}))$ is the connective complex K-theory of the manifold $X(\mathbf{C})$.

(ii) Let $\pi \in R$ be a uniformizer of $R$, and let $B$ be any $R$-dagger or affinoid algebra. Then $(\pi) \subseteq B$ is topologically nilpotent. Calvo [Cal85] proved that the reduction $B \rightarrow B /(\pi)$ induces an isomorphism $K_{\text {top }}^{-i}(B) \stackrel{\cong}{\rightarrow} K_{\text {top }}^{-i}(B /(\pi))$. This last group is the Karoubi-Villamayor K-theory of $B /(\pi)$. In particular, if $B /(\pi)$ is regular, this coincides with the Quillen K-theory: $K_{\text {top }}^{-i}(B) \stackrel{\cong}{\rightarrow}$ $K_{i}(B /(\pi))$ [Ger73, 3.14].

(iii) If, in the situation of the definition, $\pi$ is invertible on $X=\operatorname{Spec}(A)$, i.e., the special fibre $X_{k}$ is empty, then the completion $\widehat{A}$ is the zero ring, and the topological K-theory of $X$ vanishes. 
3.3 (Connection with algebraic K-theory). Recall that, for any ring $A$, the Karoubi-Villamayor K-groups [KV71] can be defined as

$$
K V_{i}(A)=\pi_{i}\left(B \cdot \operatorname{GL}\left(A\left[\Delta^{\bullet}\right]\right)\right), \quad i \geqslant 1,
$$

where $A\left[\Delta^{\bullet}\right]$ is the simplicial ring with $A\left[\Delta^{n}\right]=A\left[x_{0}, \ldots, x_{n}\right] /\left(\sum_{i} x_{i}-1\right)$. There is a natural map from the Quillen K-group $K_{i}(A)$ to $K V_{i}(A)$, which is an isomorphism when $A$ is regular. Since we are only interested in the case of regular rings, we will in the following identify $K_{i}(A)=K V_{i}(A)$.

Consider a smooth affine $R$-scheme $X=\operatorname{Spec}(A)$, as above. There is a natural map $A\left[\Delta^{\bullet}\right] \rightarrow A^{\dagger}\left\langle\Delta^{\bullet}\right\rangle^{\dagger}$. We define the bisimplicial set

$$
F(X):=F(A):=B_{\bullet} \operatorname{GL}\left(A\left[\Delta^{\bullet}\right]\right) \times_{B_{\bullet} \mathrm{GL}\left(A^{\dagger}\left\langle\Delta^{\bullet}\right\rangle^{\dagger}\right)} E_{\bullet} \operatorname{GL}\left(A^{\dagger}\left\langle\Delta^{\bullet}\right\rangle^{\dagger}\right)
$$

and the relative $K$-groups of $X$

$$
K_{i}^{\mathrm{rel}}(X):=\pi_{i} F(A), \quad i>0 .
$$

We will also need the following finite level variant of $F(A)$ :

$$
F_{r}(A):=B_{\bullet} \operatorname{GL}_{r}\left(A\left[\Delta^{\bullet}\right]\right) \times_{B_{\bullet} \mathrm{GL}_{r}\left(A^{\dagger}\left\langle\Delta^{\bullet}\right\rangle^{\dagger}\right)} E_{\bullet} \mathrm{GL}_{r}\left(A^{\dagger}\left\langle\Delta^{\bullet}\right\rangle^{\dagger}\right),
$$

so $F(A)=\lim _{\rightarrow} F_{r}(A)$ and $K_{i}^{\mathrm{rel}}(X)=\lim _{r} \pi_{i}\left(F_{r}(A)\right)$. Since the projection $E_{\bullet} \mathrm{GL}\left(A^{\dagger}\left\langle\Delta^{\bullet}\right\rangle^{\dagger}\right) \rightarrow B . \mathrm{GL}\left(A^{\dagger}\left\langle\Delta^{\bullet}\right\rangle^{\dagger}\right)$ is a Kan fibration on the diagonal simplicial sets, and since $E_{\bullet} \operatorname{GL}\left(A^{\dagger}\left\langle\Delta^{\bullet}\right\rangle^{\dagger}\right)$ is contractible, we get the following.

LEMMA 3.6. There are long exact sequences

$$
\cdots \rightarrow K_{i}^{\mathrm{rel}}(X) \rightarrow K_{i}(X) \rightarrow K_{\mathrm{top}}^{-i}(X) \rightarrow K_{i-1}^{\mathrm{rel}}(X) \rightarrow \cdots
$$

In the following, we will use the notation $\operatorname{GL}(X):=\mathrm{GL}\left(\Gamma\left(X, \mathscr{O}_{X}\right)\right)$, where $X$ can be a scheme, a dagger space, or a weak formal scheme.

We extend the definition of topological and relative K-theory to smooth separated $R$-schemes $X$ of finite type as follows. Write $\Delta_{R}^{\bullet}:=\operatorname{Spec}\left(R\left[\Delta^{\bullet}\right]\right)$. Since K-theory for regular schemes satisfies Zariski descent, we have isomorphisms

$$
K_{i}(X) \cong \underset{U_{\bullet} \rightarrow X}{\lim _{[}} \pi_{i} \operatorname{holim}_{[q] \in \Delta}\left(B_{\bullet} \operatorname{GL}\left(U_{q} \times_{R} \Delta_{R}^{\bullet}\right)\right), \quad i \geqslant 1
$$

where $U_{\bullet} \rightarrow X$ runs through all finite affine open coverings of $X$ viewed as simplicial schemes (see [Hub95, Proposition 18.1.5]). In analogy to (3.7), we define

$$
K_{\text {top }}^{-i}(X):=\underset{U_{\bullet} \rightarrow X}{\lim _{[q] \in \Delta}} \pi_{i} \operatorname{holim}_{[q]} \operatorname{GL}\left(\widehat{U}_{q} \times_{R} \widehat{\Delta}_{R}^{\bullet}\right), \quad i \geqslant 1,
$$


and

$$
K_{i}^{\mathrm{rel}}(X):=\underset{U_{\bullet} \rightarrow X}{\lim } \pi_{i} \operatorname{holim}_{[q] \in \Delta} F\left(U_{q}\right) .
$$

Here, $\widehat{\Delta}_{R}^{p}$ is the weak completion of the algebraic standard simplex $\Delta_{R}^{p}$, so if $U=\operatorname{Spec}(A)$, then $\widehat{U} \times_{R} \widehat{\Delta}_{R}^{p}=\operatorname{Spwf}\left(A^{\dagger} \otimes_{R}^{\dagger} R\left\langle\Delta^{p}\right\rangle^{\dagger}\right)$ (see 2.1). These groups are contravariantly functorial in $X$.

LEMMA 3.8. (i) If $X=\operatorname{Spec}(A)$ is affine and smooth over $R$, these definitions coincide with the earlier ones.

(ii) If $X$ is a smooth separated $R$-scheme of finite type, there is an isomorphism $K_{\text {top }}^{-i}(X) \cong K_{i}\left(X_{k}\right)$.

(iii) There are long exact sequences

$$
\cdots \rightarrow K_{i}^{\mathrm{rel}}(X) \rightarrow K_{i}(X) \rightarrow K_{\mathrm{top}}^{-i}(X) \rightarrow K_{i-1}^{\mathrm{rel}}(X) \rightarrow \cdots,
$$

as before.

Proof. (ii) Calvo's theorem 3.2(ii) implies that for a smooth affine $R$-scheme $U$ we have weak equivalences

$$
\text { B. GL }\left(\widehat{U} \times_{R} \widehat{\Delta}_{R}^{\bullet}\right) \stackrel{\sim}{\rightarrow} B . \operatorname{GL}\left(U_{k} \times_{k} \Delta_{k}^{\bullet}\right) .
$$

Since holim preserves weak equivalences between fibrant simplicial sets, for any open affine covering $U_{\bullet} \rightarrow X$, viewed as a simplicial scheme, we get weak equivalences

$$
\underset{[q] \in \Delta}{\operatorname{holim}} B . \operatorname{GL}\left(\widehat{U}_{q} \times_{R} \widehat{\Delta}_{R}^{\bullet}\right) \stackrel{\sim}{\rightarrow} \operatorname{holim}_{[q] \in \Delta} B . \operatorname{GL}\left(\left(U_{q}\right)_{k} \times_{k} \Delta_{k}^{\bullet}\right) .
$$

Taking $\pi_{i}$ and the limit over all finite affine coverings yields isomorphisms

$$
\begin{aligned}
\underset{U_{\bullet \rightarrow X} \rightarrow}{\lim _{[q] \in \Delta}} \pi_{i} \operatorname{holim}_{\bullet} B L\left(\widehat{U}_{q} \times_{R} \widehat{\Delta}_{R}^{\bullet}\right) & \stackrel{\cong}{\underset{U_{\bullet} \rightarrow X}{\rightrightarrows}} \lim _{i} \operatorname{holim}_{[q] \in \Delta} B \cdot \operatorname{GL}\left(\left(U_{q}\right)_{k} \times_{k} \Delta_{k}^{\bullet}\right) \\
& \stackrel{(3.7)}{\cong} K_{i}\left(X_{k}\right) .
\end{aligned}
$$

This proves (ii). Using Calvo's result $K_{\text {top }}^{-i}\left(A^{\dagger}\right) \cong K_{i}\left(X_{k}\right)$, we get (i) for $K_{\text {top }}^{-*}$. Using the five lemma, the result for $K_{*}^{\text {rel }}$ follows from this, (3.7) again, and (iii).

(iii) follows from Lemma 3.6 and the fact that holim preserves homotopy fibrations of fibrant simplicial sets [Tho85, Lemma 5.12]. 
REMARK 3.9. Using $K$-dagger or $K$-affinoid algebras in the above constructions, we get a notion of topological $\mathrm{K}$-theory for rigid $K$-spaces. It is likely that this coincides with the one defined by Ayoub [Ayo] using the stable homotopy categories of schemes and of rigid varieties, respectively.

\section{Preliminaries on functorial complexes}

For our construction of the relative Chern character we need functorial complexes computing the different cohomology theories involved. The main work has been done before, by Huber [Hub95], Besser [Bes00b], and Chiarellotto et al. [CCM13]. The only difference in our approach is the systematic use of dagger spaces, also initiated by Huber and Kings [HK11], which simplifies the construction of the rigid and syntomic complexes.

4.1 (Godement resolutions). We recall some facts on Godement resolutions (see [CCM13, Sections 3 and 4] for more details and references). To a morphism of sites $u: P \rightarrow X$ and an abelian sheaf $\mathcal{F}$ on $X$ one associates a cosimplicial sheaf $[p] \mapsto\left(u_{*} u^{*}\right)^{p+1} \mathcal{F}$ on $X$, where the structure maps are induced by the unit and the counit of the adjoint pair $\left(u^{*}, u_{*}\right)$ between the categories of abelian sheaves on $P$ and $X$. The associated complex of sheaves on $X$ will be denoted by $\operatorname{Gd}_{P} \mathcal{F}$. There is a canonical augmentation $\mathcal{F} \rightarrow \operatorname{Gd}_{P} \mathcal{F}$, which is a quasi-isomorphism if $u^{*}$ is exact and conservative (see, e.g., [Ivo05, Lemma 3.4.1]).

We want to use this in the situation where $P$ is a certain set of points of (the topos associated with) $X$ with the discrete topology. The first case is that of a scheme $X$. Here, we take $P=\operatorname{Pt}(X)$ to be the set of all points of the underlying topological space of $X$. Then $u^{*}$ is given by $\mathcal{F} \mapsto \bigsqcup_{x \in X} \mathcal{F}_{x}$, which is exact and conservative, and $\mathcal{F} \stackrel{\widetilde{\sim}}{\rightarrow} \operatorname{Gd}_{P t(X)} \mathcal{F}$ is the usual Godement resolution.

The second case is that of a dagger space $X$. Here, it is not enough to take just the usual points of $X$. Instead, one has to use the set of prime filters on $X$ (introduced in [vdPS95]), as alluded to in [Bes00b] and carried out in [CCM13, Section 3]. We take $P=P t(X)$ to be the set of prime filters ([CCM13, Ex. 3.2.3]) on the rigid space $X^{\text {rig }}$ associated with $X$ with the discrete topology. Then there are morphisms of sites $\operatorname{Pt}(X) \underset{[\mathrm{CCM13,3.2.3]}}{\stackrel{\xi}{\longrightarrow}} X^{\text {rig }} \underset{\text { see } 2.2}{\stackrel{u}{\longrightarrow}} X$.

Since $u$ is the identity on underlying G-topological spaces, we get from [CCM13, Lemma 3.2.5] that $\xi^{*} u^{*}$ is exact and conservative. Hence, for any abelian sheaf $\mathcal{F}$ on $X$, the augmentation $\mathcal{F} \rightarrow \operatorname{Gd}_{P t(X)} X$ is a quasi-isomorphism.

It is important to note that in both cases the complex $\operatorname{Gd}_{P} \mathcal{F}$ consists of flabby sheaves. This follows automatically, since on a discrete site every sheaf is flabby, and direct images of flabby sheaves are flabby. 
More generally, if $\mathcal{F}^{*}$ is a bounded below complex of abelian sheaves on $X$, we can apply $\mathrm{Gd}_{P}$ to each component to get a double complex. We then denote by $\operatorname{Gd}_{P} \mathcal{F}^{*}$ its associated total complex. It follows from a simple spectral sequence argument that the induced morphism $\mathcal{F}^{*} \rightarrow \mathrm{Gd}_{P} \mathcal{F}^{*}$ is a quasi-isomorphism.

An important feature of the Godement resolution is its functorial behaviour. If

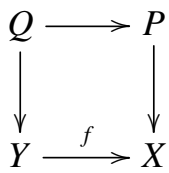

is a commutative diagram of sites, and $\mathcal{F}$ (respectively, $\mathcal{G}$ ) is a sheaf on $X$ (respectively, $Y$ ), then a morphism $\mathcal{F} \rightarrow f_{*} \mathcal{G}$ induces a morphism $\operatorname{Gd}_{P} \mathcal{F} \rightarrow$ $f_{*} \operatorname{Gd}_{Q} \mathcal{G}$ compatible with the augmentations [CCM13, Lemma 3.1.2].

4.2 (Analytic de Rham cohomology). Let $X$ be a smooth $K$-dagger space. There is a notion of differential forms on $X$ (see [GK00, §4]): for an open affinoid $U=\operatorname{Sp}(A)$, the differential $d: A=\Gamma\left(U, \mathscr{O}_{X}\right) \rightarrow \Gamma\left(U, \Omega_{X / K}^{1}\right)$ is universal for $K$-derivations of $A$ in finite $A$-modules. As usual, one constructs the de Rham complex $\Omega_{X / K}^{*}$ and defines

$$
H_{\mathrm{dR}}^{*}(X / K):=\mathbb{H}^{*}\left(X, \Omega_{X / K}^{*}\right) .
$$

We define a complex of $K$-vector spaces, functorial in the $K$-dagger space $X$ :

$$
R \Gamma_{\mathrm{dR}}(X / K):=\Gamma\left(X, \operatorname{Gd}_{P t(X)} \Omega_{X / K}^{*}\right) .
$$

Since the complex $\operatorname{Gd}_{P t(X)} \Omega_{X / K}^{*}$ consists of flabby sheaves, which are acyclic for $\Gamma(X$, . ), we have natural isomorphisms

$$
H^{*}\left(R \Gamma_{\mathrm{dR}}(X / K)\right) \cong H_{\mathrm{dR}}^{*}(X / K) .
$$

4.3 (Algebraic de Rham cohomology). Here, $X$ is a smooth separated scheme of finite type over $K$. Its de Rham cohomology is by definition the hypercohomology of the complex of Kähler differential forms:

$$
H_{\mathrm{dR}}^{*}(X / K):=\mathbb{H}^{*}\left(X, \Omega_{X / K}^{*}\right) .
$$

It is equipped with the Hodge filtration constructed as follows. By Nagata's compactification theorem and Hironaka's resolution of singularities, there exists a good compactification of $X$, i.e., an open immersion $X \stackrel{j}{\hookrightarrow} \bar{X}$ of $X$ in a smooth proper $K$-scheme $\bar{X}$ such that the complement $D=\bar{X}-X$ is a divisor with 
normal crossings. On $\bar{X}$ one has the complex $\Omega_{\bar{X} / K}^{*}(\log D)$ of differential forms with logarithmic poles along $D$. There are isomorphisms

$$
H_{\mathrm{dR}}^{*}(X / K) \cong \mathbb{H}^{*}\left(\bar{X}, \Omega_{\bar{X} / K}^{*}(\log D)\right),
$$

the Hodge-de Rham spectral sequence $E_{1}^{p, q}=H^{q}\left(\bar{X}, \Omega_{\bar{X} / K}^{p}(\log D)\right) \Rightarrow$ $H_{\mathrm{dR}}^{*}(X / K)$ degenerates at $E_{1}$, and the induced filtration $F^{\bullet} H_{\mathrm{dR}}^{*}(X / K)$ is independent of the choice of $\bar{X}$. It is given by

$$
F^{n} H_{\mathrm{dR}}^{*}(X / K)=\mathbb{H}^{*}\left(\bar{X}, \Omega_{\bar{X} / K}^{\geqslant n}(\log D)\right),
$$

$\geqslant n$ denoting the naive truncation.

If $f: X \rightarrow Y$ is a morphism of smooth separated $K$-schemes of finite type, one can construct good compactifications $X \hookrightarrow \bar{X}, Y \hookrightarrow \bar{Y}$ such that $f$ extends to a morphism $\bar{f}: \bar{X} \rightarrow \bar{Y}$. This implies that the Hodge filtration is functorial. Moreover, the induced map $f^{*}: H_{\mathrm{dR}}^{*}(Y / K) \rightarrow H_{\mathrm{dR}}^{*}(X / K)$ is strict, i.e., $f^{*}\left(F^{i} H_{\mathrm{dR}}^{*}(Y / K)\right)=F^{i} H_{\mathrm{dR}}^{*}(X / K) \cap \operatorname{im}\left(f^{*}\right)$. Indeed, by the Lefschetz principle and Serre's GAGA, this follows from the corresponding fact over $\mathbf{C}$, proven by Deligne [Del71, Théorème 3.2.5].

Since the good compactifications of $X$ form a directed set with respect to maps under $X$, and taking the colimit along a directed set is exact, to get functorial complexes computing algebraic de Rham cohomology together with its Hodge filtration we could take the colimit of the $\Gamma\left(\bar{X}, \operatorname{Gd}_{P t(\bar{X})} \Omega_{\bar{X} / K}^{*}(\log D)\right)$ along the system of good compactifications $\bar{X}$ of $X$. However, for the comparison with analytic de Rham cohomology, it is technically easier to use the following variant (see [CCM13, Proposition 4.2.3]).

Let $X^{\dagger}$ be the dagger analytification of $X$ (see 2.2), and let $\operatorname{Pt}\left(X^{\dagger}\right)$ be as in 4.1. Let $\operatorname{Pt}(\bar{X})$ be the usual set of points of the good compactification $\bar{X}$ of $X$. We can form the disjoint sum $\operatorname{Pt}\left(X^{\dagger}\right) \sqcup \operatorname{Pt}(\bar{X})$ viewed as a site with the discrete topology to get a commutative diagram of sites:

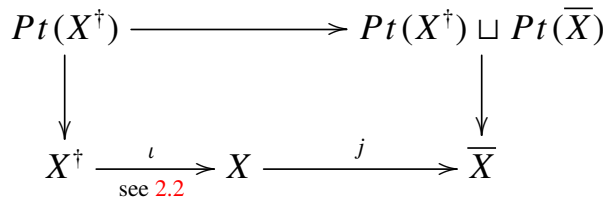

There are natural morphisms $\Omega_{\bar{X} / K}^{*}(\log D) \rightarrow j_{*} \Omega_{X / K}^{*} \rightarrow j_{*} \iota_{*} \Omega_{X^{\dagger} / K}^{*}$ which together with (4.4) induce a natural map

$$
\operatorname{Gd}_{P t\left(X^{\dagger}\right) \sqcup P t(\bar{X})} \Omega_{\bar{X} / K}^{*}(\log D) \rightarrow j_{*} \iota_{*} \operatorname{Gd}_{P t\left(X^{\dagger}\right)} \Omega_{X^{\dagger} / K}^{*} \cdot
$$


Thus we are led to define

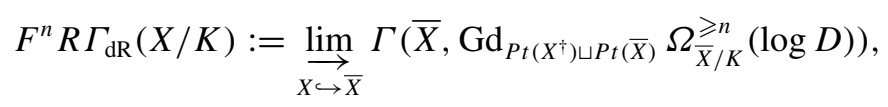

where the limit runs over the directed set of good compactifications of $X$.

It follows from the discussion above that there are natural isomorphisms

$$
H^{*}\left(F^{n} R \Gamma_{\mathrm{dR}}(X / K)\right) \cong F^{n} H_{\mathrm{dR}}^{*}(X / K),
$$

and (4.5) induces natural comparison maps

$$
F^{n} R \Gamma_{\mathrm{dR}}(X / K) \rightarrow R \Gamma_{\mathrm{dR}}\left(X^{\dagger} / K\right) .
$$

\section{The relative Chern character}

As before, $R$ denotes a complete discrete valuation ring with field of fractions $K$ of characteristic 0 and residue field $k$ of characteristic $p>0$. Let $\mathrm{Sm}_{R}$ be the category of smooth separated $R$-schemes of finite type. For $X \in \mathrm{Sm}_{R}$, we have its generic fibre $X_{K}$ with dagger analytification $X_{K}^{\dagger}$, and its weak completion $\widehat{X}$ with generic fibre $\widehat{X}_{K}$, related by the following morphisms of locally G-ringed spaces:

$$
\widehat{X}_{K} \subseteq X_{K}^{\dagger} \stackrel{\iota}{\rightarrow} X_{K}
$$

In particular, we have morphisms of complexes

$$
F^{n} R \Gamma_{\mathrm{dR}}\left(X_{K} / K\right) \stackrel{(4.7)}{\longrightarrow} R \Gamma_{\mathrm{dR}}\left(X_{K}^{\dagger} / K\right) \stackrel{\text { by functoriality }}{\longrightarrow} R \Gamma_{\mathrm{dR}}\left(\widehat{X}_{K} / K\right) .
$$

5.2. We denote by $\mathrm{Ch}$ the category of complexes of abelian groups. For a morphism $A \stackrel{f}{\rightarrow} B$ in Ch, we denote by $\operatorname{mf}(A \rightarrow B):=\operatorname{Cone}(A \rightarrow B)[-1]$ the mapping fibre. It has the following property: if $C$ is a complex, the morphisms $C \rightarrow \operatorname{mf}(A \rightarrow B)$ are in one-to-one correspondence with pairs $(g, h)$, where $g: C \rightarrow A$ is a morphism of complexes and $h: C \rightarrow B[-1]$ is a homotopy such that $d h+h d=f \circ g$.

DEFINITION 2. For every integer $n$, we define a functor $R \Gamma_{\text {rel }}(., n): \mathrm{Sm}_{R}^{\mathrm{op}} \rightarrow$ Ch by

$$
R \Gamma_{\text {rel }}(X, n):=\operatorname{mf}\left(F^{n} R \Gamma_{\mathrm{dR}}\left(X_{K} / K\right) \stackrel{(5.1)}{\longrightarrow} R \Gamma_{\mathrm{dR}}\left(\widehat{X}_{K} / K\right)\right)
$$

and relative cohomology groups $H_{\mathrm{rel}}^{*}(X, n):=H^{*}\left(R \Gamma_{\mathrm{rel}}(X, n)\right)$.

REMARKS 5.3. (i) These are closely related to rigid syntomic cohomology; see Lemma 6.9 below. The complex $R \Gamma_{\text {rel }}(X, n)$ can also be interpreted as the syntomic $P$-complex $\mathbb{R} \Gamma_{\mathrm{f}, 1}(X, n)$ of [Bes00a, 2.2] for the polynomial $P=1$. 
(ii) Since $X / R$ is smooth, the de Rham cohomology of $\widehat{X}_{K}$ is just the rigid cohomology of the special fibre $X_{k}$ (see Section 6.1 below). Hence the relative cohomology groups sit in exact sequences

$$
\cdots \rightarrow H_{\mathrm{rel}}^{i}(X, n) \rightarrow F^{n} H_{\mathrm{dR}}^{i}\left(X_{K} / K\right) \rightarrow H_{\mathrm{rig}}^{i}\left(X_{k} / K\right) \rightarrow \cdots .
$$

(iii) If $X / R$ is proper, then $\widehat{X}_{K}=X_{K}^{\dagger}$ (see 2.2), and by GAGA [GK99, Kor. 4.5] $R \Gamma_{\mathrm{dR}}\left(X_{K}^{\dagger} / K\right) \simeq R \Gamma_{\mathrm{dR}}\left(X_{K} / K\right)$, where $\simeq$ denotes a quasi-isomorphism. Hence $R \Gamma_{\text {rel }}(X, n) \simeq \operatorname{mf}\left(F^{n} R \Gamma_{\mathrm{dR}}\left(X_{K} / K\right) \rightarrow R \Gamma_{\mathrm{dR}}\left(X_{K} / K\right)\right)$ in this case, and the degeneration of the Hodge-de Rham spectral sequence yields isomorphisms

$$
H_{\mathrm{rel}}^{i}(X, n) \cong H_{\mathrm{dR}}^{i-1}\left(X_{K} / K\right) / F^{n} H_{\mathrm{dR}}^{i-1}\left(X_{K} / K\right) .
$$

(iv) For an interpretation of the relative cohomology in terms of stable $\mathbf{A}^{1}$-homotopy theory, we refer the reader to [DM12].

The goal of this section is to construct relative Chern character maps which will be homomorphisms

$$
\operatorname{ch}_{n, i}^{\mathrm{rel}}: K_{i}^{\mathrm{rel}}(X) \rightarrow H_{\mathrm{rel}}^{2 n-i}(X, n) .
$$

We first describe an abstract formalism to obtain homomorphisms from the homotopy groups of certain simplicial sets to the cohomology of suitable functorial complexes, and then specialize this to the construction of the relative Chern character and, in the next section, of the syntomic regulator. This formalization of the constructions makes it easier to compare them afterwards.

5.4. We view complexes in Ch either homologically $\cdots \rightarrow C_{i} \stackrel{d}{\rightarrow} C_{i-1} \rightarrow \cdots$ or cohomologically $\cdots \rightarrow C^{-i} \stackrel{d}{\rightarrow} C^{-i+1} \rightarrow \cdots$, using the convention $C_{i}=C^{-i}$. Given $A, B \in \mathrm{Ch}$, we denote by $\operatorname{Hom}(A, B)$ the mapping complex. In degree $i$, it is given by $\prod_{p} \operatorname{Hom}\left(A^{p}, B^{p+i}\right)$ with differential $f \mapsto f \circ d^{A}-(-1)^{i} d^{B} \circ f$. In particular, cycles in degree $i$ are given by $Z^{i} \underline{\operatorname{Hom}}(A, B)=\operatorname{Hom}_{\mathrm{Ch}}(A, B[i])$. If $C^{\bullet, \bullet}$ is a double complex, the differential of the total complex is given on $C^{p, q}$ by $d^{\text {horiz }}+(-1)^{p} d^{\text {vert }}$ ( $p$ is the horizontal coordinate).

5.5. We consider the following set-up: $\mathrm{S}$ is a category, $a: \mathrm{Sm}_{R} \rightarrow \mathrm{S}$ a functor, $\Gamma_{0}: \mathrm{Sm}_{R}^{\mathrm{op}} \rightarrow \mathrm{Ch}$ and $\Gamma_{1}: \mathrm{S}^{\mathrm{op}} \rightarrow \mathrm{Ch}$ are functorial complexes, and we have a natural transformation $\Gamma_{0} \rightarrow \Gamma_{1} \circ a$.

For example, $\mathrm{S}$ could be the category of smooth weak formal $R$-schemes $\mathrm{Smf}_{R}, a: \mathrm{Sm}_{R} \rightarrow \mathrm{Smf}_{R}$ the weak completion functor $X \mapsto \widehat{X}, \Gamma_{0}$ the functorial 
complex $F^{n} R \Gamma_{\mathrm{dR}}\left((.)_{K} / K\right), \Gamma_{1}=R \Gamma_{\mathrm{dR}}\left((.)_{K} / K\right)$, and $\Gamma_{0} \rightarrow \Gamma_{1} \circ a$ the natural transformation given by (5.1).

We fix a morphism $E \rightarrow B$ in $\mathrm{Sm}_{R}$. In applications, this will typically be the morphism of simplicial schemes $E$. GL $r, R \rightarrow B$. GL $r, R$. Consider a map $X \stackrel{f}{\rightarrow} B$ in $\mathrm{Sm}_{R}$ together with a map $a(X) \stackrel{g}{\rightarrow} a(E)$ in $\mathrm{S}$ such that

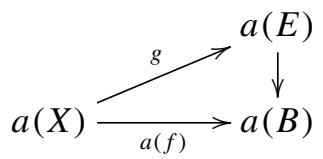

commutes; in other words, $(f, g)$ is an element in $B(X) \times_{a(B)(a(X))} a(E)(a(X))$, where we use the notation $B(X):=\operatorname{Hom}_{\mathrm{Sm}}(X, B)$, etc. By abuse of notation, we write $\left(B \times_{a(B)} a(E)\right)(X)$ for this set. Then the pair $(f, g)$ gives a commutative diagram

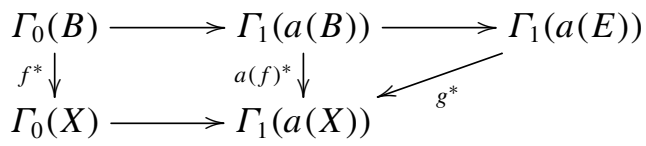

in Ch, and hence a morphism of complexes, i.e., a zero cycle in the Hom-complex,

$$
\operatorname{mf}\left(\Gamma_{0}(B) \rightarrow \Gamma_{1}(a(E))\right) \rightarrow \operatorname{mf}\left(\Gamma_{0}(X) \rightarrow \Gamma_{1}(a(X))\right) .
$$

This construction induces a morphism of complexes

$$
\begin{aligned}
\mathbf{Z}[( & \left.\left(B \times_{a(B)} a(E)\right)(X)\right] \\
& \rightarrow \underline{\operatorname{Hom}}\left(\operatorname{mf}\left(\Gamma_{0}(B) \rightarrow \Gamma_{1}(a(E))\right), \operatorname{mf}\left(\Gamma_{0}(X) \rightarrow \Gamma_{1}(a(X))\right)\right),
\end{aligned}
$$

where $\mathbf{Z}[$. ] is the free abelian group considered as a complex in degree 0 .

If $E_{\bullet} \rightarrow B_{\bullet}$ is a morphism of simplicial objects in $\mathrm{Sm}_{R}$, and $X^{\bullet}$ is a cosimplicial object in $\mathrm{Sm}_{R}$, then $([p],[q]) \mapsto\left(B_{p} \times_{a\left(B_{p}\right)} a\left(E_{p}\right)\right)\left(X^{q}\right)$ is a bisimplicial set, and we get a natural map of complexes

$$
\begin{aligned}
\operatorname{Tot} \mathbf{Z}\left[\left(B_{\bullet} \times_{a\left(B_{\bullet}\right)} a\left(E_{\bullet}\right)\right)\left(X^{\bullet}\right)\right] & \\
& \rightarrow \underline{\operatorname{Hom}}\left(\operatorname{mf}\left(\Gamma_{0}\left(B_{\bullet}\right) \rightarrow \Gamma_{1}\left(a\left(E_{\bullet}\right)\right)\right), \operatorname{mf}\left(\Gamma_{0}\left(X^{\bullet}\right) \rightarrow \Gamma_{1}\left(a\left(X^{\bullet}\right)\right)\right)\right) .
\end{aligned}
$$

Here, $\Gamma_{0}\left(B_{\bullet}\right), \Gamma_{0}\left(X^{\bullet}\right)$, etc., are defined as the direct sum total complexes, and we view the simplicial (respectively, cosimplicial) direction as the horizontal one. For example, the degree $n$-component $\left(\Gamma_{0}\left(X^{\bullet}\right)\right)^{n}$ of the total complex is the possibly infinite direct sum $\bigoplus_{p, q \in \mathbf{Z}, p+q=n} \Gamma_{0}^{q}\left(X^{-p}\right)$. On the left-hand side, the vertical direction is that coming from $X^{\bullet}$. Using the sign conventions from 5.4, we have to 
introduce a sign $(-1)^{q(q-1) / 2}$ in bidegree $(p, q)$ in order that (5.6) is a morphism of complexes.

On homology, (5.6) induces for every integer $*$ a map

$$
\begin{aligned}
& H_{i}\left(\operatorname{Tot} \mathbf{Z}\left[\left(B_{\bullet} \times_{a\left(B_{\bullet}\right)} a\left(E_{\bullet}\right)\right)\left(X^{\bullet}\right)\right]\right) \\
& \rightarrow \operatorname{Hom}\left(H^{*}\left(\operatorname{mf}\left(\Gamma_{0}\left(B_{\bullet}\right) \rightarrow \Gamma_{1}\left(a\left(E_{\bullet}\right)\right)\right)\right), H^{*-i}\left(\operatorname { m f } \left(\Gamma_{0}\left(X^{\bullet}\right)\right.\right.\right. \\
& \left.\left.\left.\quad \rightarrow \Gamma_{1}\left(a\left(X^{\bullet}\right)\right)\right)\right)\right) .
\end{aligned}
$$

In particular, any class $c \in H^{2 n}\left(\operatorname{mf}\left(\Gamma_{0}\left(B_{\bullet}\right) \rightarrow \Gamma_{1}\left(a\left(E_{\bullet}\right)\right)\right)\right)$ gives by composing (5.7) with the evaluation at $c$ a map

$$
\begin{aligned}
{ }^{*} c: & H_{i}\left(\operatorname{Tot} \mathbf{Z}\left[\left(B_{\bullet} \times_{a\left(B_{\bullet}\right.} a\left(E_{\bullet}\right)\right)\left(X^{\bullet}\right)\right]\right) \\
& \rightarrow H^{2 n-i}\left(\operatorname{mf}\left(\Gamma_{0}\left(X^{\bullet}\right) \rightarrow \Gamma_{1}\left(a\left(X^{\bullet}\right)\right)\right)\right) .
\end{aligned}
$$

DEFINITION 3. A regulator datum is a tuple $\omega$ consisting of (1) a category $\mathrm{S}$ together with a functor $a: \mathrm{Sm}_{R} \rightarrow \mathrm{S}$, (2) functors $\Gamma_{0}: \mathrm{Sm}_{R}^{\mathrm{op}} \rightarrow \mathrm{Ch}, \Gamma_{1}: \mathrm{S}^{\text {op }} \rightarrow$ Ch together with a natural transformation $\Gamma_{0} \rightarrow \Gamma_{1} \circ a$, (3) a morphism of simplicial objects $E_{\bullet} \rightarrow B_{\bullet}$ in $\mathrm{Sm}_{R}$, and (4) a class $c \in H^{2 n}\left(\operatorname{mf}\left(\Gamma_{0}\left(B_{\bullet}\right) \rightarrow\right.\right.$ $\left.\left.\Gamma_{1}\left(a\left(E_{\bullet}\right)\right)\right)\right)$.

To simplify notation, we denote such a regulator datum by $\omega=\left(\mathrm{S}, E_{\bullet} \rightarrow B_{\bullet}\right.$, $\left.\Gamma_{0} \rightarrow \Gamma_{1} \circ a, c\right)$.

LEMMA 5.9. A regulator datum $\omega$ induces for every cosimplicial object $X^{\bullet}$ in $\mathrm{Sm}_{R}$ and $i \geqslant 0$ a homomorphism

$$
\operatorname{reg}_{i}(\omega): \pi_{i}\left(\left(B_{\bullet} \times_{a\left(B_{\bullet}\right)} a\left(E_{\bullet}\right)\right)\left(X^{\bullet}\right)\right) \rightarrow H^{2 n-i}\left(\operatorname{mf}\left(\Gamma_{0}\left(X^{\bullet}\right) \rightarrow \Gamma_{1}\left(a\left(X^{\bullet}\right)\right)\right)\right) .
$$

Proof. The desired homomorphism $\operatorname{reg}_{i}(\omega)$ is the composition

$$
\begin{aligned}
& \pi_{i}\left(\left(B_{\bullet} \times_{a\left(B_{\bullet}\right)} a\left(E_{\bullet}\right)\right)\left(X^{\bullet}\right)\right) & & \\
\longrightarrow & H_{i}\left(\mathbf{Z}\left[\operatorname{diag}\left(B_{\bullet} \times_{a\left(B_{\bullet}\right)} a\left(E_{\bullet}\right)\right)\left(X^{\bullet}\right)\right]\right) & & \text { Hurewicz } \\
\cong & H_{i}\left(\operatorname{Tot} \mathbf{Z}\left[\left(B_{\bullet} \times_{a\left(B_{\bullet}\right)} a\left(E_{\bullet}\right)\right)\left(X^{\bullet}\right)\right]\right) & & \text { by Eilenberg-Zilber } \\
\stackrel{{ }_{c} c}{\longrightarrow} & H^{2 n-i}\left(\operatorname{mf}\left(\Gamma_{0}\left(X^{\bullet}\right) \rightarrow \Gamma_{1}\left(a\left(X^{\bullet}\right)\right)\right)\right) & & \text { by }(5.8) .
\end{aligned}
$$

We record the following naturality properties which are easily established. They will be used in the comparison of the relative with the syntomic Chern character in the next section. Consider two regulator data $\omega=\left(\mathrm{S}, E_{\bullet} \rightarrow B_{\bullet}, \Gamma_{0} \rightarrow \Gamma_{1} \circ a, c\right)$ and $\omega^{\prime}=\left(\mathrm{S}^{\prime}, E_{\bullet} \rightarrow B_{\bullet}, \Gamma_{0}^{\prime} \rightarrow \Gamma_{1}^{\prime} \circ a^{\prime}, c^{\prime}\right)$ with the same $E_{\bullet} \rightarrow B_{\bullet}$.

Assume, moreover, that we have a functor $b: \mathrm{S} \rightarrow \mathrm{S}^{\prime}$ such that $b \circ a \cong a^{\prime}$, natural transformations $\Gamma_{0} \rightarrow \Gamma_{0}^{\prime}, \Gamma_{1} \rightarrow \Gamma_{1}^{\prime} \circ b$, and a natural homotopy $h$ between 
the compositions $\Gamma_{0} \rightarrow \Gamma_{0}^{\prime} \rightarrow \Gamma_{1}^{\prime} \circ a^{\prime}$ and $\Gamma_{0} \rightarrow \Gamma_{1} \circ a \rightarrow \Gamma_{1}^{\prime} \circ b \circ a \cong \Gamma_{1}^{\prime} \circ a^{\prime}$. For every map $Z \rightarrow Y$ in $\operatorname{Sm}_{R}$, these induce a map $\operatorname{mf}\left(\Gamma_{0}(Y) \rightarrow \Gamma_{1}(a(Z))\right) \rightarrow$ $\operatorname{mf}\left(\Gamma_{0}^{\prime}(Y) \rightarrow \Gamma_{1}^{\prime}\left(a^{\prime}(Z)\right)\right)$ (see 5.2).

LEMMA 5.10. If $c$ maps to $c^{\prime}$ by the map $H^{2 n}\left(\operatorname{mf}\left(\Gamma_{0}\left(B_{\bullet}\right) \rightarrow \Gamma_{1}\left(a\left(E_{\bullet}\right)\right)\right)\right) \rightarrow$ $H^{2 n}\left(\operatorname{mf}\left(\Gamma_{0}^{\prime}\left(B_{\bullet}\right) \rightarrow \Gamma_{1}^{\prime}\left(a^{\prime}\left(E_{\bullet}\right)\right)\right)\right)$, then for every $X^{\bullet}$ the diagram

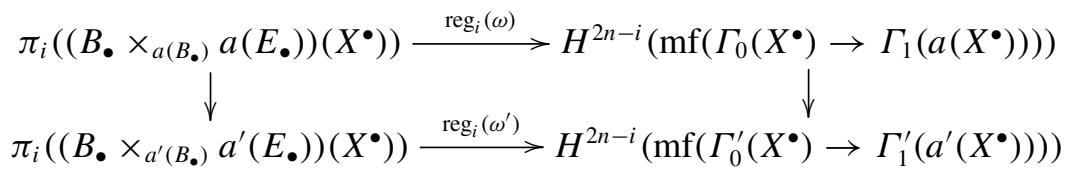

commutes.

We now consider regulator data $\omega=\left(\mathrm{S}, E_{\bullet} \rightarrow B_{\bullet}, \Gamma_{0} \rightarrow \Gamma_{1} \circ a, c\right)$ and $\omega^{\prime}=\left(\mathrm{S}, E_{\bullet}^{\prime} \rightarrow B_{\bullet}^{\prime}, \Gamma_{0} \rightarrow \Gamma_{1} \circ a, c^{\prime}\right)$ with the same category $\mathrm{S}$ and complexes $\Gamma_{0}, \Gamma_{1}$, and assume that we have a commutative diagram of simplicial objects in $\mathrm{Sm}_{R}$ :

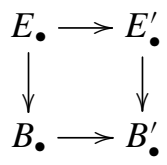

LEMMA 5.11. If $c^{\prime} \in H^{2 n}\left(\operatorname{mf}\left(\Gamma_{0}\left(B_{\bullet}^{\prime}\right) \rightarrow \Gamma_{1}\left(a\left(E_{\bullet}^{\prime}\right)\right)\right)\right)$ maps to the class $c \in$ $H^{2 n}\left(\operatorname{mf}\left(\Gamma_{0}\left(B_{\bullet}\right) \rightarrow \Gamma_{1}\left(a\left(E_{\bullet}\right)\right)\right)\right)$ by the induced map, then for every $X^{\bullet}$ the diagram

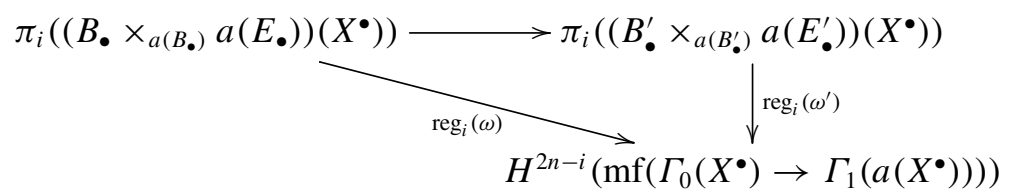

commutes.

We now construct a regulator datum $\omega_{n,(r)}^{\text {rel }}$ that produces the relative Chern character. Let $\mathrm{Smf}_{R}$ be the category of smooth weak formal $R$-schemes. Let $a: \mathrm{Sm}_{R} \rightarrow \mathrm{Smf}_{R}$ be the weak completion functor $X \mapsto \widehat{X}$, let $\Gamma_{0}$ be given by $X \mapsto F^{n} R \Gamma_{\mathrm{dR}}\left(X_{K} / K\right)$, let $\Gamma_{1}$ be given by $\mathscr{X} \mapsto R \Gamma_{\mathrm{dR}}\left(\mathscr{X}_{K} / K\right)$, and let the natural transformation $\Gamma_{0} \rightarrow \Gamma_{1} \circ a$ be given by (5.1). For $E_{\bullet} \rightarrow B_{\bullet}$, we take $E_{.} \mathrm{GL}_{r} \rightarrow B_{\bullet} \mathrm{GL}_{r}$. As cosimplicial object in $\mathrm{Sm}_{R}$ we will always take $X^{\bullet}=X \times_{R} \Delta_{R}^{\bullet}=\operatorname{Spec}\left(A\left[\Delta^{\bullet}\right]\right)$ for some affine $X=\operatorname{Spec}(A)$ in $\operatorname{Sm}_{R}$. 
With these choices we have

$$
\begin{array}{ll}
\left(B_{\bullet} \times_{a\left(B_{\bullet}\right)} a\left(E_{\bullet}\right)\right)\left(X^{\bullet}\right)=F_{r}(A), & \text { see }(3.5), \\
\operatorname{mf}\left(\Gamma_{0}\left(X^{\bullet}\right) \rightarrow \Gamma_{1}\left(a\left(X^{\bullet}\right)\right)\right)=R \Gamma_{\text {rel }}\left(X \times_{R} \Delta_{R}^{\bullet}, n\right) & \text { by Definition } 2 .
\end{array}
$$

For the equality (5.12) we use that $\widehat{\mathrm{GL}}_{r}(\widehat{X})=\operatorname{Hom}_{\mathrm{Smf}_{R}}\left(\widehat{X}, \widehat{\mathrm{GL}}_{r}\right) \cong \mathrm{GL}_{r}\left(A^{\dagger}\right)$. To get a regulator datum, we need to specify the class $c$. This is accomplished by the following.

LEMMA 5.14. For each $r \geqslant 1$, the natural map

$$
\begin{aligned}
& H^{2 n}\left(\operatorname{mf}\left(F^{n} R \Gamma_{\mathrm{dR}}\left(B \cdot \mathrm{GL}_{r, K} / K\right) \rightarrow R \Gamma_{\mathrm{dR}}\left(E \cdot \widehat{\mathrm{GL}}_{r, K} / K\right)\right)\right) \\
& \quad \rightarrow H^{2 n}\left(F^{n} R \Gamma_{\mathrm{dR}}\left(B \cdot \mathrm{GL}_{r, K} / K\right)\right)=F^{n} H_{\mathrm{dR}}^{2 n}\left(B . \mathrm{GL}_{r, K} / K\right)
\end{aligned}
$$

is an isomorphism.

Proof. This follows from the long exact sequence for the cohomology of a cone together with the fact that $E_{\bullet} \widehat{\mathrm{GL}}_{r, K}$ is a contractible simplicial dagger space, and hence has no cohomology in positive degrees (see [Tam10, Lemma 2.11]).

In particular, there is a unique class

$$
\operatorname{ch}_{n,(r)}^{\mathrm{rel}} \in H^{2 n}\left(\operatorname{mf}\left(F^{n} R \Gamma_{\mathrm{dR}}\left(B \cdot \mathrm{GL}_{r, K} / K\right) \rightarrow R \Gamma_{\mathrm{dR}}\left(E_{\bullet} \widehat{\mathrm{GL}}_{r, K} / K\right)\right)\right)
$$

which is mapped to the degree $2 n$ component $\operatorname{ch}_{n,(r)}^{\mathrm{dR}} \in F^{n} H_{\mathrm{dR}}^{2 n}\left(B . \mathrm{GL}_{r, K} / K\right)$ of the universal Chern character class in de Rham cohomology. Since the $\mathrm{ch}_{n,(r)}^{\mathrm{dR}}$ are compatible for varying $r \geqslant 1$, so are the $\mathrm{ch}_{n,(r)}^{\text {syn }}$.

We also need the following lemma. At this point it is crucial to work with dagger spaces.

LEMMA 5.16. The natural map $R \Gamma_{\text {rel }}(X, n) \rightarrow R \Gamma_{\text {rel }}\left(X \times \widehat{\Delta}_{R}^{\bullet}, n\right)$ is a quasiisomorphism for any $X \in \mathrm{Sm}_{R}$.

Proof. It suffices to check this for both components of the cone separately. We first show that $R \Gamma_{\mathrm{dR}}\left(\widehat{X}_{K} / K\right) \rightarrow R \Gamma_{\mathrm{dR}}\left(\widehat{X}_{K} \times \widehat{\Delta}_{K}^{\bullet} / K\right)$ is a quasi-isomorphism.

By construction, $R \Gamma_{\mathrm{dR}}\left(\widehat{X}_{K} \times \widehat{\Delta}_{K}^{\bullet} / K\right)$ is the direct sum total complex of a double complex in the second quadrant. The filtration by columns gives a convergent spectral sequence in the second quadrant (see [Wei94, 5.6.1] for the dual homological case),

$$
E_{1}^{p, q}=H_{\mathrm{dR}}^{q}\left(\widehat{X}_{K} \times \widehat{\Delta}_{K}^{-p} / K\right) \Rightarrow H^{p+q}\left(R \Gamma_{\mathrm{dR}}\left(\widehat{X}_{K} \times \widehat{\Delta}_{K}^{\bullet} / K\right)\right) .
$$


The differential $d_{1}$ is induced from the cosimplicial structure of $\widehat{\Delta}_{K}^{\bullet}$. The homotopy invariance of de Rham cohomology of dagger spaces [GK99, Proposition 5.8] implies that $d_{1}^{p, q}$ is the identity if $p$ is even and zero if $p$ is odd. Hence $E_{2}^{0, q}=H_{\mathrm{dR}}^{q}\left(\widehat{X}_{K} / K\right), E_{2}^{p, q}=0$ if $p<0$. It follows that the edge morphism $E_{2}^{0, q}=H_{\mathrm{dR}}^{q}\left(\widehat{X}_{K} / K\right) \rightarrow H^{q}\left(R \Gamma_{\mathrm{dR}}\left(\widehat{X}_{K} \times \widehat{\Delta}_{K}^{\bullet} / K\right)\right)$ is an isomorphism.

For $F^{n} R \Gamma_{\mathrm{dR}}\left(X_{K} / K\right) \rightarrow F^{n} R \Gamma_{\mathrm{dR}}\left(X_{K} \times \Delta_{K}^{\bullet} / K\right)$ we argue similarly, using in addition that $H_{\mathrm{dR}}^{*}\left(X_{K} / K\right) \rightarrow H_{\mathrm{dR}}^{*}\left(X_{K} \times_{K} \Delta_{K}^{-p} / K\right)$ is an isomorphism by the homotopy invariance of de Rham cohomology; hence so is $F^{n} H_{\mathrm{dR}}^{*}\left(X_{K} / K\right) \stackrel{\cong}{\rightarrow}$ $F^{n} H_{\mathrm{dR}}^{*}\left(X_{K} \times_{K} \Delta_{K}^{-p} / K\right)$ by strictness (see 4.3).

Definition 4. Let $X=\operatorname{Spec}(A)$ be a smooth affine $R$-scheme of finite type. Let $\omega_{n,(r)}^{\text {rel }}$ be the regulator datum $\left(\mathrm{Smf}_{R}, E \cdot \mathrm{GL}_{r} \rightarrow B \cdot \mathrm{GL}_{r}, F^{n} R \Gamma_{\mathrm{dR}}\left((.)_{K} / K\right) \rightarrow\right.$ $\left.R \Gamma_{\mathrm{dR}}\left(()_{K} / K\right), \mathrm{ch}_{n,(r)}^{\mathrm{rel}}\right)$. By 5.9, this gives homomorphisms

$$
\operatorname{reg}_{i}\left(\omega_{n,(r)}^{\mathrm{rel}}\right): \pi_{i}\left(F_{r}(A)\right) \rightarrow H^{2 n-i}\left(R \Gamma_{\mathrm{rel}}\left(X \times \Delta^{\bullet}, n\right)\right) \stackrel{5.14}{\cong} H_{\mathrm{rel}}^{2 n-i}(X, n),
$$

which are compatible for varying $r \geqslant 1$. The relative Chern character is defined to be the colimit

$$
\operatorname{ch}_{n, i}^{\mathrm{rel}}: K_{i}^{\mathrm{rel}}(X)=\underset{r}{\lim _{r}} \pi_{i}\left(F_{r}(A)\right) \stackrel{\lim _{r} \mathrm{reg}_{i}\left(\omega_{n,(r)}^{\mathrm{rel}}\right)}{\longrightarrow} H_{\mathrm{rel}}^{2 n-i}(X, n) .
$$

We use Jouanolou's trick to extend this definition to all schemes in $\mathrm{Sm}_{R}$. According to Jouanolou and Thomason [Wei89, 4.4], such a scheme $X$ admits a Jouanolou torsor $W \stackrel{p}{\rightarrow} X$, i.e., $W$ is affine and $p$ is a torsor for some vector bundle on $X$.

LEMMA 5.17. In the above situation, the map $p^{*}: R \Gamma_{\text {rel }}(X, n) \rightarrow R \Gamma_{\text {rel }}(W, n)$ is a quasi-isomorphism.

Proof. It is enough to show that $p$ induces a quasi-isomorphism on both components of the cone. We show that $R \Gamma_{\mathrm{dR}}\left(\widehat{X}_{K} / K\right) \stackrel{p^{*}}{\rightarrow} R \Gamma_{\mathrm{dR}}\left(\widehat{W}_{K} / K\right)$ is a quasi-isomorphism. Using again that $p^{*}: H_{\mathrm{dR}}^{*}\left(X_{K} / K\right) \rightarrow H_{\mathrm{dR}}^{*}\left(W_{K} / K\right)$ is strict with respect to the Hodge filtrations (see 4.3), the proof for $F^{n} R \Gamma_{\mathrm{dR}}$ is similar.

Choose a finite open covering $X=\bigcup_{\alpha \in A} U_{\alpha}$ such that $p^{-1}\left(U_{\alpha}\right) \rightarrow U_{\alpha}$ is isomorphic to a trivial vector bundle $\mathbb{A}_{U_{\alpha}}^{r} \rightarrow U_{\alpha}$. Let $U_{\bullet} \rightarrow X$ be the Čech nerve of this covering, and denote by $p^{-1}\left(U_{\bullet}\right) \rightarrow W$ its base change to $W$. Since $\left\{\left(\widehat{U}_{\alpha}\right)_{K}\right\}_{\alpha \in A}$ is an admissible covering of $\widehat{X}_{K}$, it follows that $R \Gamma_{\mathrm{dR}}\left(\widehat{X}_{K} / K\right) \rightarrow$ $R \Gamma_{\mathrm{dR}}\left(\left(\widehat{U}_{\bullet}\right)_{K} / K\right)$ and similarly $\left.R \Gamma_{\mathrm{dR}}\left(\widehat{W}_{K} / K\right) \rightarrow R \Gamma_{\mathrm{dR}}\left(\widehat{p^{-1}\left(U_{\bullet}\right.}\right)_{K} / K\right)$ are quasiisomorphisms. Hence we are reduced to the case that $W \rightarrow X$ is of the form 
$\mathbb{A}_{X}^{r} \rightarrow X$. Then the claim follows from homotopy invariance for the de Rham cohomology of dagger spaces [GK99, Proposition 5.8]

Definition 5. Let $X$ be in $\mathrm{Sm}_{R}$, and choose a Jouanolou torsor $W \stackrel{p}{\rightarrow} X$. We define the relative Chern character to be the composition

$$
K_{i}^{\mathrm{rel}}(X) \stackrel{p^{*}}{\rightarrow} K_{i}^{\mathrm{rel}}(W) \stackrel{\mathrm{ch} \mathrm{ch}_{n, i}^{\mathrm{rel}}}{\longrightarrow} H_{\mathrm{rel}}^{2 n-i}(W, n) \stackrel{\left(p^{*}\right)^{-1}}{\cong} H_{\mathrm{rel}}^{2 n-i}(X, n) .
$$

One checks that this does not depend on the choice of $W \rightarrow X$ using the fact that, for two Jouanolou torsors $W \rightarrow X, W^{\prime} \rightarrow X$, the fibre product $W \times_{X} W^{\prime} \rightarrow$ $X$ is again a Jouanolou torsor.

\section{Comparison with the rigid syntomic regulator}

The main technical problem in the construction of the rigid syntomic regulator is the construction of functorial complexes computing rigid and rigid syntomic cohomology. This was solved by Besser [Bes00b]. An alternative construction of the regulator using cycle classes and higher Chow groups instead of K-theory is given in [CCM13]. We recall Besser's construction with some improvements from [CCM13]. As in [HK11], the systematic use of dagger spaces simplifies the construction a little bit.

Let $R$ be as before, and assume moreover that the residue field $k$ of $R$ is perfect. Let $K_{0} \subseteq K$ be the field of fractions of the ring of Witt vectors of $k$.

6.1. Rigid cohomology. We consider the category $\mathrm{Sch}_{k}$ of separated schemes of finite type over $k$ which admit a closed immersion in a flat weakly formal $R$ scheme $\mathscr{Y}$ with smooth special fibre $\mathscr{Y}_{k}$. For $X \in \mathrm{Sm}_{R}$, the special fibre $X_{k}$ is in $\mathrm{Sch}_{k}$, as we can take the closed immersion of $X_{k}$ in the weak completion $\widehat{X}$ of $X$. Let $X$ be in $\mathrm{Sch}_{k}$, and choose an embedding $X \hookrightarrow \mathscr{Y}$ as above. The rigid cohomology of $X$ with coefficients in $K$ is by definition the de Rham cohomology of the tube $] X\left[\mathscr{Y}:=\operatorname{sp}^{-1}(X) \subset \mathscr{Y}_{K}\right.$ (see 2.2) of $X$ in $\mathscr{Y}$ :

$$
H_{\text {rig }}^{*}(X / K)=H_{\mathrm{dR}}^{*}(] X[\mathscr{Y} / K)
$$

[Ber97], [GK99, Proposition 8.1]. Up to isomorphism this is independent of the choice of $\mathscr{Y}$.

Following Besser, we define

$$
R \Gamma_{\text {rig }}(X / K)_{\mathscr{Y}}:=R \Gamma_{\mathrm{dR}}(] X[\mathscr{Y} / K) .
$$


This complex is functorial only in the pair $(X, \mathscr{Y})$. To obtain complexes functorial in the $k$-scheme $X$, we proceed as in [Bes00b, §4]. Define the category of rigid pairs $\mathcal{R} \mathcal{P}$ : objects are pairs $(X, j: X \hookrightarrow \mathscr{Y})$, where $X$ and $j$ are as above. We will often abbreviate such a pair as $(X, \mathscr{Y})$. Morphisms $\left(X^{\prime}, \mathscr{Y}^{\prime}\right) \rightarrow(X, \mathscr{Y})$ are pairs of morphisms $\left(f: X^{\prime} \rightarrow X, F:\right] X^{\prime}\left[\mathscr{Y}^{\prime} \rightarrow\right] X[\mathscr{Y})$ such that the diagram

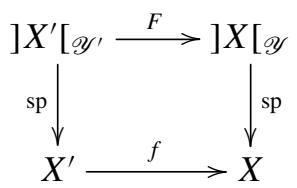

commutes. The category $\mathcal{R} \mathcal{P}$ substitutes Besser's category $\mathcal{R} \mathcal{T}$ of rigid triples. Note that there is a natural functor $\mathrm{Sm}_{R} \rightarrow \mathcal{R} \mathcal{P}$ taking $X$ to the pair $\left(X_{k}, \widehat{X}\right)$.

With this replacement, Besser's construction [Bes00b, Proposition 4.9, Corollary 4.22] goes through word by word and yields the following.

Proposition 6.1. (i) There exists a functor $R \Gamma_{\text {rig }}(., / k): \operatorname{Sch}_{k}^{\text {op }} \rightarrow \mathrm{Ch}_{K}$ to the category of complexes of $K$-vector spaces such that $H^{*}\left(R \Gamma_{\text {rig }}(X / K)\right)=$ $H_{\text {rig }}^{*}(X / K)$ functorially. If $K$ is absolutely unramified, i.e., $K=K_{0}$, and $\sigma$ is the Frobenius on $K_{0}$, there exists a natural $\sigma$-semilinear Frobeniusendomorphism $\phi$ on $R \Gamma_{\text {rig }}\left(X / K_{0}\right)$.

(ii) There exists a functor $\mathcal{R} \mathcal{P}^{\mathrm{op}} \rightarrow \mathrm{Ch}_{K},(X, \mathscr{Y}) \mapsto \widetilde{R \Gamma}$ rig $(X / K)_{\mathscr{Y}}$ together with $\mathcal{R} \mathcal{P}$-functorial quasi-isomorphisms

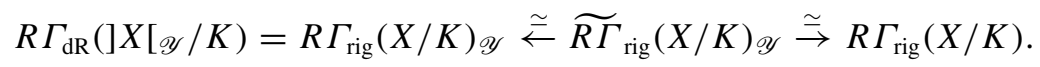

6.2. Rigid syntomic cohomology. Recall that the homotopy pullback of a diagram of complexes $A \stackrel{f}{\rightarrow} C \stackrel{g}{\leftarrow} B$ is by definition the complex $A \tilde{\times}_{C} B:=$ Cone $(A \oplus B \stackrel{f-g}{\longrightarrow} C)[-1]$. It fits in a diagram

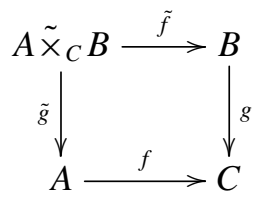

which is commutative up to canonical homotopy, given by the projection to the $C$-component of the cone. If $f$ is a quasi-isomorphism, so is $\tilde{f}$. 
6.2. Let $X$ be in $\mathrm{Sm}_{R}$. Then $] X_{k}\left[\widehat{X}=\widehat{X}_{K} \subseteq X_{K}^{\dagger}\right.$, and we have natural maps of complexes, functorial in $X$,

$$
F^{n} R \Gamma_{\mathrm{dR}}\left(X_{K} / K\right) \stackrel{(5.1)}{\longrightarrow} R \Gamma_{\mathrm{dR}}(] X_{k}[\widehat{X} / K) \stackrel{\simeq}{\widetilde{R}} \widetilde{R} \text { rig }\left(X_{k} / K\right)_{\widehat{X}} \stackrel{\simeq}{\rightarrow} R \Gamma_{\text {rig }}\left(X_{k} / K\right) .
$$

Define $F^{n} \widetilde{R \Gamma}$ dR $(X / K)$ to be the homotopy pullback of the left two arrows above, a complex quasi-isomorphic to $F^{n} R \Gamma_{\mathrm{dR}}\left(X_{K} / K\right)$, which admits a natural map

$$
F^{n} \widetilde{R \Gamma}_{\mathrm{dR}}(X / K) \rightarrow \widetilde{R \Gamma} \widetilde{\text { rig }}_{k}\left(X_{k} / K\right)_{\widehat{X}}
$$

On $R \Gamma_{\text {rig }}\left(X_{k} / K_{0}\right)$, we have the Frobenius $\phi$ and the natural map to $R \Gamma_{\text {rig }}\left(X_{k} / K\right)$. We define the complex

$$
\Phi(n)\left(X_{k}\right):=\operatorname{Cone}\left(R \Gamma_{\text {rig }}\left(X_{k} / K_{0}\right) \rightarrow R \Gamma_{\text {rig }}\left(X_{k} / K_{0}\right) \oplus R \Gamma_{\text {rig }}\left(X_{k} / K\right)\right),
$$

where the map is given by $\omega \mapsto\left(\left(1-\left(\phi / p^{n}\right)\right) \omega, \omega\right)$. This complex is functorial in the $k$-scheme $X_{k}$, and there are natural maps

$$
\widetilde{R \Gamma}_{\text {rig }}\left(X_{k} / K\right)_{\widehat{X}} \stackrel{\simeq}{\rightarrow} R \Gamma_{\text {rig }}\left(X_{k} / K\right) \rightarrow \Phi(n)\left(X_{k}\right) .
$$

Consider the composition

$$
F^{n} \widetilde{R \Gamma}_{\mathrm{dR}}(X / K) \stackrel{(6.3)}{\longrightarrow} \widetilde{R \Gamma}_{\mathrm{rig}}\left(X_{k} / K\right)_{\widehat{X}} \stackrel{(6.4)}{\longrightarrow} \Phi(n)\left(X_{k}\right) .
$$

DEFINITION 6. We define the syntomic complex of $X$ twisted by $n$ :

$$
R \Gamma_{\mathrm{syn}}(X, n):=\operatorname{mf}\left(F^{n} \widetilde{R \Gamma} \mathrm{dR}(X / K) \stackrel{-(6.5)}{\longrightarrow} \Phi(n)\left(X_{k}\right)\right) .
$$

Its cohomology groups will be denoted by $H_{\text {syn }}^{*}(X, n)$.

REMARK 6.6. Writing down the iterated cone construction explicitly, one sees that $R \Gamma_{\text {syn }}(X, n)$ is isomorphic to the complex

$\operatorname{Cone}\left(R \Gamma_{\text {rig }}\left(X_{k} / K_{0}\right) \oplus F^{n} \widetilde{R \Gamma}_{\mathrm{dR}}(X / K) \rightarrow R \Gamma_{\text {rig }}\left(X_{k} / K_{0}\right) \oplus R \Gamma_{\text {rig }}\left(X_{k} / K\right)\right)[-1]$, where the map is given by $(x, y) \mapsto\left(\left(1-\left(\phi / p^{n}\right)\right) x, x-y\right)$. From this one sees that the fundamental Proposition 6.3 of [Bes00b] also holds for our definition of rigid syntomic cohomology. Hence all further constructions of [Bes00b] work equally well in our setting. In particular, there are natural maps

$$
H_{\text {syn }}^{*}(X, n) \rightarrow F^{n} H_{\mathrm{dR}}^{*}\left(X_{K} / K\right) .
$$

In fact, it is possible to construct a natural chain of quasi-isomorphisms connecting our version of the rigid syntomic complex with Besser's. But since we do not need this we omit the lengthy and technical details. 
We now give Besser's construction of the rigid syntomic regulator [Bes00b, Theorem 7.5] in the set-up of the present paper. By [Bes00b, Proposition 7.4] and the discussion following it, for all positive integers $n, r$ there exists a class

$$
\operatorname{ch}_{n,(r)}^{\mathrm{syn}} \in H_{\mathrm{syn}}^{2 n}\left(B . \mathrm{GL}_{r, R}, n\right),
$$

the universal $n$th syntomic Chern character class, uniquely determined by the fact that it is mapped to the degree $2 n$ component $\operatorname{ch}_{n,(r)}^{\mathrm{dR}}$ of the universal de Rham Chern character class in $F^{n} H_{\mathrm{dR}}^{2 n}\left(B_{\bullet} \mathrm{GL}_{r, K} / K\right)$ under (6.7). These are compatible for varying $r$.

Let $a=(.)_{k}: \mathrm{Sm}_{R} \rightarrow \mathrm{Sch}_{k}$ be the special fibre functor. We define the syntomic regulator datum

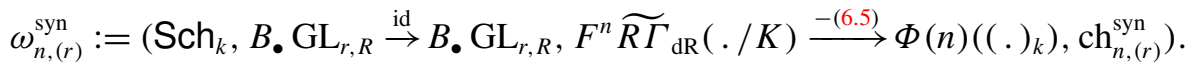

Now, let $X=\operatorname{Spec}(A)$ be an affine scheme in $\mathrm{Sm}_{R}$, and as always $X^{\bullet}=X \times_{R}$ $\Delta_{R}^{\bullet}$. With these choices, we have

$$
\begin{aligned}
& \left(B . \mathrm{GL}_{r, R} \times_{a\left(B \bullet \mathrm{GL}_{r, R}\right)} a\left(B \bullet \mathrm{GL}_{r, R}\right)\right)\left(X^{\bullet}\right)=B . \mathrm{GL}_{r, R}\left(A\left[\Delta^{\bullet}\right]\right) \text {, } \\
& \operatorname{mf}\left(F^{n} \widetilde{R \Gamma} \mathrm{dR}\left(X^{\bullet}\right) \rightarrow \Phi(n)\left(X_{k}^{\bullet}\right)\right)=R \Gamma_{\text {syn }}\left(X \times_{R} \Delta_{R}^{\bullet}, n\right), \\
& \operatorname{mf}\left(F^{n} \widetilde{R \Gamma}{ }_{\mathrm{dR}}\left(B . \mathrm{GL}_{r, R}\right) \rightarrow \Phi(n)\left(B_{\bullet} \mathrm{GL}_{r, k}\right)\right)=R \Gamma_{\mathrm{syn}}\left(B_{\bullet} \mathrm{GL}_{r, R}, n\right) .
\end{aligned}
$$

Similarly as in Lemma 5.16, one shows that $R \Gamma_{\text {syn }}(X, n) \rightarrow R \Gamma_{\text {syn }}\left(X \times_{R} \Delta_{R}^{\bullet}, n\right)$ is a quasi-isomorphism.

DEFINITION 7. For $i \geqslant 1$, the syntomic Chern character or regulator is given by

$$
\begin{gathered}
\operatorname{ch}_{n, i}^{\mathrm{syn}}: K_{i}(X)=\underset{r}{\lim _{r}} \pi_{i}\left(B . G L_{r}\left(A\left[\Delta^{\bullet}\right]\right)\right) \stackrel{\lim _{r} \operatorname{reg}_{i}\left(\omega_{n,(r)}^{\mathrm{syn}}\right)}{\longrightarrow} \\
H^{2 n-i}\left(R \Gamma_{\mathrm{syn}}\left(X \times_{R} \Delta_{R}^{\bullet}, n\right)\right) \cong H_{\mathrm{syn}}^{2 n-i}(X, n) .
\end{gathered}
$$

Using that the natural map $B_{\bullet} \mathrm{GL}(A) \rightarrow \operatorname{diag} B_{\bullet} \mathrm{GL}\left(A\left[\Delta^{\bullet}\right]\right)$ induces an isomorphism in homology with $\mathbf{Z}$-coefficients (see the proof of Lemma 7.14), it is easy to check that this construction is equivalent to Besser's in the affine case.

Again, this is extended to all schemes in $\mathrm{Sm}_{R}$ using Jouanolou's trick (see Definition 5). 


\subsection{The comparison.}

LEMMA 6.9. There exist complexes $\widetilde{R \Gamma}$ rel $(X, n)$, functorial in $X \in \mathrm{Sm}_{R}$, together with maps

$$
R \Gamma_{\text {rel }}(X, n) \stackrel{\simeq}{\longleftarrow} \widetilde{R \Gamma}_{\text {rel }}(X, n) \rightarrow R \Gamma_{\text {syn }}(X, n),
$$

the left-pointing arrow being a quasi-isomorphism. These induce natural maps

$$
H_{\mathrm{rel}}^{*}(X, n) \rightarrow H_{\mathrm{syn}}^{*}(X, n)
$$

which are isomorphisms if $X$ is proper and $* \notin\{2 n, 2 n+1,2 n+2\}$.

Proof. Consider the following diagram of complexes:

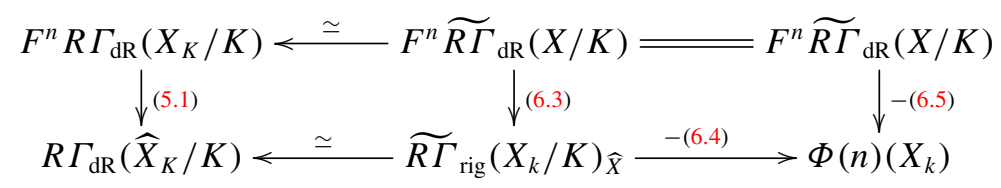

The left square commutes up to canonical homotopy (see 6.2); the right square strictly commutes. We set $\widetilde{R \Gamma}$ rel $(X, n):=\operatorname{mf}\left(F^{n} \widetilde{R \Gamma}_{\mathrm{dR}}(X / K) \rightarrow\right.$ $\left.\widehat{R} \Gamma_{\text {rig }}\left(X_{k} / K\right)_{\widehat{X}}\right)$. The desired maps are induced by the maps in the diagram together with the homotopy which makes the left-hand square commute (see 5.2).

The second statement follows from weight considerations, as in the proof of [Bes00b, Proposition 8.6].

We can now formulate the main result of this paper.

THEOREM 6.12. For every $X$ in $\mathrm{Sm}_{R}$ and $i \geqslant 1$, the diagram

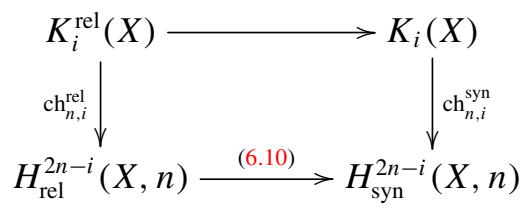

commutes.

Proof. By construction of the maps in the diagram, we may suppose that $X=\operatorname{Spec}(A)$ is affine. Write $A_{k}=A \otimes_{R} k$, so $X_{k}$ is isomorphic to $\operatorname{Spec}\left(A_{k}\right)$. 
We split the diagram up into the following smaller diagrams, and show that every single one of them commutes.

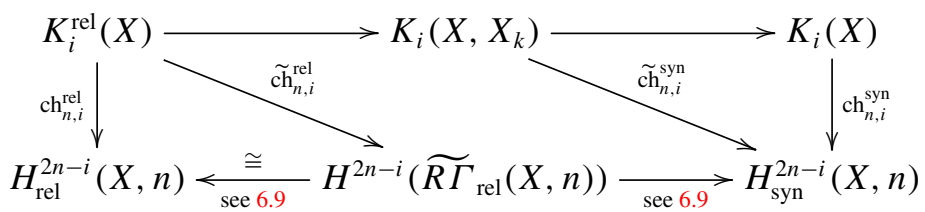

Here, $K_{i}\left(X, X_{k}\right):=\pi_{i}\left(B_{\bullet} \operatorname{GL}\left(A\left[\Delta^{\bullet}\right]\right) \times_{B_{\bullet} G L\left(A_{k}\left[\Delta^{\bullet}\right]\right)} E_{\bullet} \operatorname{GL}\left(A_{k}\left[\Delta^{\bullet}\right]\right)\right)$. Since $A_{k}\left[\Delta^{\bullet}\right] \cong A^{\dagger}\left\langle\Delta^{\bullet}\right\rangle^{\dagger} \otimes_{R} k$, the map $K_{i}^{\text {rel }}(X) \rightarrow K_{i}(X)$ factors through $K_{i}\left(X, X_{k}\right)$.

All vertical (respectively, diagonal) maps are induced by a compatible family of maps for each finite level $r$. We can thus restrict consideration to a fixed finite level $r$. To ease notation, we write $E_{\bullet}:=E_{\bullet} \mathrm{GL}_{r, R}, B_{\bullet}:=B_{\bullet} \mathrm{GL}_{r, R}$. The diagonal maps arise as follows: since the left-hand square in (6.11) commutes up to canonical homotopy, we are in the situation of Lemma 5.10. In particular, we have a natural isomorphism (see (6.11))

$$
\begin{aligned}
& H^{2 n}\left(\operatorname{mf}\left(F^{n} \widetilde{R \Gamma}_{\mathrm{dR}}\left(B_{\bullet} / K\right) \rightarrow \widetilde{R \Gamma} \mathrm{rig}\left(E_{\bullet}, k / K\right) \widehat{E}_{\bullet}\right)\right) \\
& \quad \cong H^{2 n}\left(\operatorname{mf}\left(F^{n} R \Gamma_{\mathrm{dR}}\left(B_{\bullet} \mathrm{GL}_{r, K} / K\right) \rightarrow R \Gamma_{\mathrm{dR}}\left(E_{\bullet} \widehat{\mathrm{GL}}_{r, K} / K\right)\right)\right),
\end{aligned}
$$

and we define $\widetilde{\mathrm{ch}}_{n,(r)}^{\text {rel }}$ to be the class mapping to $\mathrm{ch}_{n,(r)}^{\text {rel }}$ (see (5.15)) under this isomorphism. We let $\widetilde{c h}_{n, i}^{\text {rel }}$ be induced by the regulator data $\widetilde{\omega}_{n,(r)}^{\text {rel }}=\left(\mathrm{Smf}_{R}, E_{\bullet} \rightarrow\right.$ $\left.B_{\bullet}, F^{n} \widetilde{R \Gamma} \mathrm{dR}(. / K) \rightarrow \widetilde{R \Gamma}_{\text {rig }}\left((.)_{k} / K\right)_{\widehat{.} .)}, \widetilde{c h}_{n,(r)}^{\text {rel }}\right)$. Then it is clear from Lemma 5.10 and the constructions that the left triangle commutes.

The map $\widetilde{c h}_{n, i}^{\text {syn }}$ is induced by the regulator datum $\widetilde{\omega}_{n,(r)}^{\text {syn }}=\left(\operatorname{Sch}_{k}, E_{\bullet} \rightarrow B_{\bullet}\right.$, $\left.F^{n} \widetilde{R \Gamma}_{\mathrm{dR}}(. / K) \stackrel{-(6.5)}{\longrightarrow} \Phi(n)\left((.)_{k}\right), \widetilde{\mathrm{ch}}_{n,(r)}^{\text {syn }}\right)$, where ${\widetilde{\mathrm{ch}_{n,(r)}}}_{n \text { sy }}$ is defined as follows. We have a commutative diagram

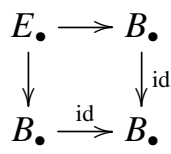

which induces a map

$$
\begin{aligned}
H_{\mathrm{syn}}^{2 n}\left(B_{\bullet}, n\right) & \cong H^{2 n}\left(\operatorname{mf}\left(F^{n} \widetilde{R \Gamma} \widetilde{\mathrm{dR}}_{(}\left(B_{\bullet} / K\right) \rightarrow \Phi(n)\left(B_{\bullet} k\right)\right)\right) \\
& \rightarrow H^{2 n}\left(\operatorname{mf}\left(F^{n} \widetilde{R \Gamma_{\mathrm{dR}}}\left(B_{\bullet} / K\right) \rightarrow \Phi(n)\left(E_{\bullet}\right)\right)\right),
\end{aligned}
$$

and $\widetilde{\mathrm{ch}}_{n,(r)}^{\text {syn }}$ is by definition the image of $\mathrm{ch}_{n,(r)}^{\text {syn }}$ (see (6.8)) by this map. It is then clear from Lemma 5.11 that the right triangle in (6.13) commutes. 
It remains to show that the middle parallelogram in (6.13) commutes. For this, we apply Lemma 5.10 to the regulator data $\widetilde{\omega}_{n}^{\text {rel }}$ and $\widetilde{\omega}_{n}^{\text {syn }}:$ the special fibre functor $\mathrm{Sm}_{R} \rightarrow \mathrm{Sch}_{k}$ factors naturally as $\mathrm{Sm}_{R} \stackrel{X \mapsto \widehat{X}}{\longrightarrow} \operatorname{Smf}_{R} \stackrel{\mathscr{X}_{\mapsto} \mathscr{X}_{k}}{\longrightarrow} \operatorname{Sch}_{k}$. Moreover, we have a natural transformation between functors $\mathrm{Smf}_{R}^{\text {op }} \rightarrow \mathrm{Ch}, \widetilde{R \Gamma}_{\text {rig }}\left((.)_{k} / K\right)_{(.)} \rightarrow$ $\Phi(n)\left((.)_{k}\right)$ given by $-(6.5)$. Since

$$
\begin{aligned}
& F^{n} \widetilde{R \Gamma}_{\mathrm{dR}}(X / K)=F^{n} \widetilde{R \Gamma}_{\mathrm{dR}}(X / K) \\
& \downarrow(6.3) \\
& \widetilde{R \Gamma}_{\text {rig }}\left(X_{k} / K\right)_{\widehat{X}} \stackrel{-(6.4)}{\longrightarrow} \Phi(n)\left(X_{k}\right)
\end{aligned}
$$

commutes for every $X \in \mathrm{Sm}_{R}$, we have a natural map

$$
\begin{gathered}
\left.H^{2 n}\left(\operatorname{mf}\left(F^{n} \widetilde{R \Gamma}_{\mathrm{dR}}\left(B_{\bullet} / K\right) \rightarrow \widetilde{R \Gamma} \widetilde{\mathrm{rig}}_{\left(E_{\bullet}, k\right.} / K\right)_{\widehat{E}_{\bullet}}\right)\right) \\
\rightarrow H^{2 n}\left(\operatorname{mf}\left(F^{n} \widetilde{R \Gamma}_{\mathrm{dR}}\left(B_{\bullet} / K\right) \rightarrow \Phi(n)\left(E_{\bullet}, k\right)\right) .\right.
\end{gathered}
$$

If we show that under this map $\widetilde{c h}_{n,(r)}^{\text {rel }}$ maps to $\widetilde{c h}_{n,(r)}^{\text {syn }}$, then Lemma 5.10 implies the desired commutativity. But, indeed, (6.14) fits in a commutative diagram

$$
\begin{aligned}
& H^{2 n}\left(\operatorname{mf}\left(F^{n} \widetilde{R \Gamma}_{\mathrm{dR}}\left(B_{\bullet} / K\right) \rightarrow \widetilde{R \Gamma}_{\mathrm{rig}}\left(E_{\bullet, k} / K\right)_{\widehat{E}_{\bullet}}\right)\right) \stackrel{\cong}{\longrightarrow} H^{2 n}\left(F^{n} \widetilde{R \Gamma}_{\mathrm{dR}}\left(B_{\bullet} / K\right)\right) \\
& { }^{(6.14)} \downarrow \\
& H^{2 n}\left(\operatorname{mf}\left(F^{n} \widetilde{R \Gamma}_{\mathrm{dR}}\left(B_{\bullet} / K\right) \rightarrow \Phi(n)\left(E_{\bullet}\right)\right)\right)
\end{aligned}
$$

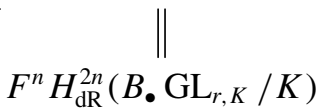

where the two isomorphisms are established as in Lemma 5.14 and, by the constructions, both $\widetilde{c h}_{n,(r)}^{\text {rel }}$ and $\widetilde{c h}_{n,(r)}^{\text {syn }}$ map to $\mathrm{ch}_{n,(r)}^{\mathrm{dR}}$ on the right-hand side.

6.4. Applications. In this section, we assume that the residue field $k$ of $R$ is finite.

Let $X$ be a smooth and proper $R$-scheme. The étale Chern character class induces a map $\operatorname{ch}_{n, i}^{\text {ét }}: K_{i}(X) \rightarrow H_{\text {ét }}^{2 n-i}\left(X_{K}, \mathbf{Q}_{p}(n)\right)$. It follows from the crystalline Weil conjectures [CLS98] and Faltings' crystalline comparison theorem [Fal89] that $H_{\text {ét }}^{2 n-i}\left(X_{\bar{K}}, \mathbf{Q}_{p}(n)\right)^{G_{K}}=0$ for $i>0$, where $\bar{K}$ denotes an algebraic closure of $K$ and $G_{K}=\operatorname{Gal}(\bar{K} / K)$. Hence the Hochschild-Serre spectral sequence for $X_{\bar{K}} \rightarrow X_{K}$ induces an edge morphism $H_{\text {ét }}^{2 n-i}\left(X_{K}, \mathbf{Q}_{p}(n)\right)=F^{1} H_{\text {ét }}^{2 n-i}\left(X_{K}\right.$, $\left.\mathbf{Q}_{p}(n)\right) \rightarrow H^{1}\left(G_{K}, H_{\mathrm{et}}^{2 n-i-1}\left(X_{\bar{K}}, \mathbf{Q}_{p}(n)\right)\right)$, and the composition

$$
r_{p}: K_{i}(X) \stackrel{\mathrm{ch}_{n, i}^{\text {ét }}}{\longrightarrow} H_{\mathrm{et}}^{2 n-i}\left(X_{K}, \mathbf{Q}_{p}(n)\right) \stackrel{\text { edge }}{\longrightarrow} H^{1}\left(G_{K}, H_{\mathrm{et}}^{2 n-i-1}\left(X_{\bar{K}}, \mathbf{Q}_{p}(n)\right)\right)
$$

is the étale p-adic regulator. 
According to the de Rham comparison theorem [Fal89], we have an isomorphism of filtered vector spaces

$$
D_{\mathrm{dR}}\left(H_{\mathrm{ett}}^{2 n-i-1}\left(X_{\bar{K}}, \mathbf{Q}_{p}(n)\right)\right) \cong H_{\mathrm{dR}}^{2 n-i-1}\left(X_{K} / K\right)(n),
$$

where the twist by $n$ on the right-hand side only shifts the filtration. Hence

$$
D_{\mathrm{dR}}\left(H_{\mathrm{et}}^{2 n-i-1}\left(X_{\bar{K}}, \mathbf{Q}_{p}(n)\right)\right) / F^{0} \cong H_{\mathrm{dR}}^{2 n-i-1}\left(X_{K} / K\right) / F^{n} \cong H_{\mathrm{rel}}^{2 n-i}(X, n)
$$

(see Remark 5.3(iii)). In particular, the Bloch-Kato exponential for the $G_{K}$-representation $H_{\mathrm{ét}}^{2 n-i-1}\left(X_{\bar{K}}, \mathbf{Q}_{p}(n)\right)$ is a map

$$
\exp : H_{\mathrm{dR}}^{2 n-i-1}\left(X_{K} / K\right) / F^{n} \rightarrow H^{1}\left(G_{K}, H_{\mathrm{ett}}^{2 n-i-1}\left(X_{\bar{K}}, \mathbf{Q}_{p}(n)\right)\right),
$$

and from Nizioł's work we get the following.

COROLlARY 6.16. For each smooth projective $R$-scheme $X$, the diagram

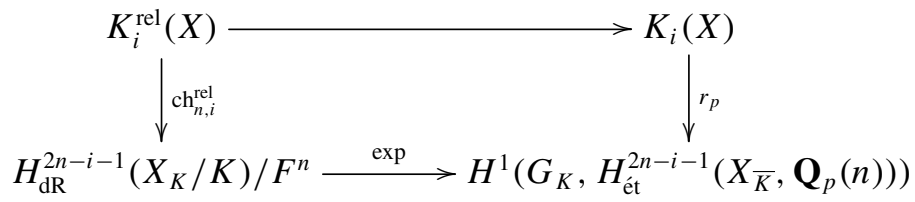

commutes.

Proof. It follows from Nizioł's work [Niz97, Niz01] and the comparison with Besser's syntomic cohomology [Bes00b, Proposition 9.9] that there is a natural map $H_{\mathrm{syn}}^{*}(X, n) \rightarrow H_{\mathrm{et}}^{*}\left(X_{K}, \mathbf{Q}_{p}(n)\right)$ which is compatible with Chern classes [Bes00b, Corollary 9.10]. By [Bes00b, Proposition 9.11], the composition

$$
\begin{aligned}
& H_{\mathrm{dR}}^{2 n-i-1}\left(X_{K} / K\right) / F^{n} \rightarrow H_{\mathrm{syn}}^{2 n-i}(X, n) \rightarrow H_{\mathrm{et}}^{2 n-i}\left(X_{K}, \mathbf{Q}_{p}(n)\right) \\
& \quad \rightarrow H^{1}\left(G_{K}, H_{\mathrm{et}}^{2 n-i-1}\left(X_{\bar{K}}, \mathbf{Q}_{p}(n)\right)\right)
\end{aligned}
$$

is the Bloch-Kato exponential for $H_{\mathrm{ét}}^{2 n-i-1}\left(X_{\bar{K}}, \mathbf{Q}_{p}(n)\right)$. Hence the claim follows from the comparison of the relative Chern character with the syntomic regulator in Theorem 6.12.

REMARK 6.17. By Calvo's result (Lemma 3.8(ii)), we have an isomorphism $K_{\text {top }}^{-i}(X) \cong K_{i}\left(X_{k}\right)$. For $X_{k}$ smooth and projective, these groups are conjectured to be torsion (Parshin's conjecture). This would imply that $K_{i}^{\text {rel }}(X) \rightarrow K_{i}(X)$ is rationally an isomorphism. It follows from [Har77] and [Qui72] that this conjecture is true for $\operatorname{dim} X_{k} \leqslant 1$. 
From the previous corollary together with our earlier work [Tam12a, Tam10], we get a new proof of the main result of [HK11]: Huber and Kings introduce the $p$-adic Borel regulator $r_{\mathrm{Bo}, p}: K_{2 n-1}(R) \rightarrow K$ by imitating the construction of the classical Borel regulator for the field of complex numbers, replacing the van Est isomorphism by the Lazard isomorphism.

Corollary 6.18 (Huber-Kings [HK11]). Let $K$ be a finite extension of $\mathbf{Q}_{p}$. The diagram

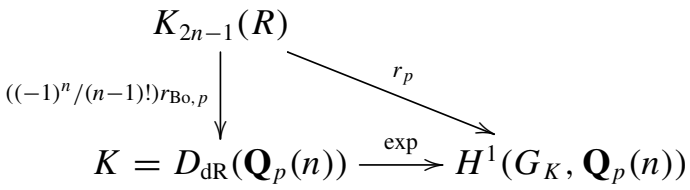

commutes.

REMARK. The factor $(-1)^{n} /(n-1)$ ! appears since Huber and Kings use Chern classes in the normalization of both the étale and the $p$-adic Borel regulator, whereas we used Chern character classes in the definition of the étale regulator.

Proof. Apply the previous corollary with $X=\operatorname{Spec}(R), i=2 n-1$. As mentioned in Remark 6.17, $K_{2 n-1}^{\text {rel }}(R) \rightarrow K_{2 n-1}(R)$ is rationally an isomorphism. In Theorem 7.20 , we will show that the present version of the relative Chern character coincides with Karoubi's original construction. For this we showed in [Tam10, Corollary 7.23] that the diagram

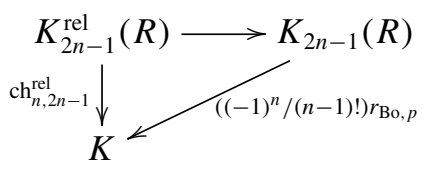

commutes.

\section{Comparison with Karoubi's original construction}

As before, $R$ denotes a complete discrete valuation ring with field of fractions $K$ of characteristic 0 and residue field $k$ of characteristic $p>0$. In this section, we compare the relative Chern character of Section 5 with Karoubi's original construction [Kar83, Kar87] in the form of [Tam10]. For any smooth affine $R$-scheme $X=\operatorname{Spec}(A)$, this is a homomorphism

$$
\operatorname{ch}_{n, i}^{\mathrm{Kar}}: K_{i}^{\mathrm{rel}}(X) \rightarrow \mathbb{H}^{2 n-i-1}\left(\widehat{X}_{K}, \Omega_{\widehat{X}_{K} / K}^{<n}\right)=H^{2 n-i-1}\left(\Omega^{<n}\left(\widehat{X}_{K}\right)\right) .
$$

We recall the main steps in its construction. 
7.1. We begin with some preliminaries concerning integration (see also [Tam12a, Appendix]). Consider the polynomial ring $\mathbf{Q}\left[\Delta^{p}\right]=\mathbf{Q}\left[x_{0}, \ldots\right.$, $\left.x_{n}\right] /\left(\sum_{i} x_{i}-1\right)$. There is a well-defined integration map $\int_{\Delta^{p}}: \Omega_{\mathbf{Q}\left[\Delta^{p}\right] / \mathbf{Q}}^{p} \rightarrow \mathbf{Q}$ sending an algebraic $p$-form $\omega$ to the integral of $\omega$, considered as a smooth $p$-form, over the real standard simplex $\boldsymbol{\Delta}^{p}:=\left\{\left(x_{0}, \ldots, x_{p}\right) \in \mathbf{R}^{p+1} \mid \sum_{i} x_{i}=1\right.$, $\left.\forall i: 0 \leqslant x_{i} \leqslant 1\right\} \subseteq \mathbf{R}^{p+1}$ with orientation given by the form $d x_{1} \cdots d x_{p}$. This integral is in fact a rational number. It satisfies Stokes's formula:

$$
\int_{\Delta^{p}} d \omega=\sum_{i=0}^{p}(-1)^{i} \int_{\Delta^{p-1}}\left(\partial^{i}\right)^{*} \omega .
$$

Similarly, one can define an integration map $\int_{\Delta^{p} \times \Delta^{q}}: \Omega_{\mathbf{Q}\left[\Delta^{p}\right] \otimes_{\mathbf{Q}} \mathbf{Q}\left[\Delta^{q}\right] / \mathbf{Q}}^{p+q} \rightarrow \mathbf{Q}$, again given by the usual integration over the product of real simplices $\boldsymbol{\Delta}^{p} \times \boldsymbol{\Delta}^{q}$. There is a canonical decomposition of $\boldsymbol{\Delta}^{p} \times \boldsymbol{\Delta}^{q}$ into copies of the standard $p+q$-simplex $\boldsymbol{\Delta}^{p+q}$, indexed by all $(p, q)$-shuffles $(\mu, v)$ (see the proof of Theorem 7.20 below). It follows from this and the analogous formula for smooth differential forms that

$$
\int_{\Delta^{p} \times \Delta^{q}} \omega=\sum_{(\mu, v)} \operatorname{sgn}(\mu, v) \int_{\Delta^{p+q}}(\mu, \nu)^{*} \omega,
$$

where the sum runs over all $(p, q)$-shuffles and $(\mu, v)^{*} \omega \in \Omega_{\mathbf{Q}\left[\Delta^{p+q}\right] / \mathbf{Q}}^{p+q}$ is the pullback of $\omega \in \Omega_{\mathbf{Q}\left[\Delta^{p}\right] \otimes_{\mathbf{Q}} \mathbf{Q}\left[\Delta^{q}\right] / \mathbf{Q}}^{p+q}$ to the simplex corresponding to the shuffle $(\mu, v)$.

Tensoring the Q-linear integration map with $K$, we get $\int_{\Delta^{p}}: \Omega_{K\left[\Delta^{p}\right] / K}^{p} \rightarrow$ $K$. More generally, for a $K$-algebra $A$, we define an integration map $\int_{\Delta^{p}}: \Omega_{A\left[\Delta^{p}\right] / K}^{n} \rightarrow \Omega_{A / K}^{n-p}$ using the decomposition $\Omega_{A\left[\Delta^{p}\right] / K}^{n} \cong \bigoplus_{k+l=n} \Omega_{A / K}^{k} \otimes_{K}$ $\Omega_{K\left[\Delta^{p}\right] / K}^{l}$.

If $A$ is a $K$-dagger algebra, one can show similarly as in [Tam12a, Appendix] that by continuity this extends uniquely to a map

$$
\int_{\Delta^{p}}: \Omega_{A\left\langle\Delta^{p}\right\rangle^{\dagger} / K, \mathrm{f}}^{n} \rightarrow \Omega_{A / K, \mathrm{f}}^{n-p},
$$

where the subscript $\mathrm{f}$ indicates that we consider differential forms for dagger algebras (see 4.2). The analogues of (7.2) and (7.3) remain valid.

7.4. Let $Y_{\bullet}$ be a simplicial dagger space. A simplicial $n$-form $\omega$ on $Y_{\bullet}$ is a collection of $n$-forms $\omega_{p}$ on $Y_{p} \times \widehat{\Delta}_{K}^{p}, p \geqslant 0$, satisfying $\left(1 \times \phi_{\Delta}\right)^{*} \omega_{p}=\left(\phi_{X} \times 1\right)^{*} \omega_{q}$ on $Y_{p} \times \widehat{\Delta}_{K}^{q}$ for all monotone maps $\phi:[q] \rightarrow[p], \phi_{\Delta}, \phi_{X}$ denoting the induced (co)simplicial structure maps. We will often denote $\omega_{p}$ by $\left.\omega\right|_{Y_{p} \times \widehat{\Delta}_{K}^{p}}$. 
The space of all simplicial $n$-forms is denoted by $D^{n}\left(Y_{\bullet}\right)$. Applying the wedge product and exterior differential componentwise makes $D^{*}\left(Y_{\bullet}\right)$ into a commutative differential graded algebra.

For a $K$-dagger space $X$, we write $\Omega^{*}(X)$ for the complex of global sections $\Gamma\left(X, \Omega_{X / K}^{*}\right)$. Define $\Omega^{*}\left(Y_{\bullet}\right)$ as the total complex of the cosimplicial complex $[p] \mapsto \Omega^{*}\left(Y_{p}\right)$. By Dupont's theorem [Tam10, Theorem 5.6],

$$
I: D^{*}\left(Y_{\bullet}\right) \rightarrow \Omega^{*}\left(Y_{\bullet}\right),\left.\quad \omega \mapsto \sum_{k} \int_{\Delta^{k}} \omega\right|_{Y_{k} \times \widehat{\Delta}_{K}^{k}}
$$

is a quasi-isomorphism.

We have a decomposition $\Omega_{X \times \widehat{\Delta}_{K}^{k} / K}^{n} \cong \bigoplus_{p+q=n} \Omega_{X \times \widehat{\Delta}_{K}^{k} / K}^{p, q}$ where $\Omega_{X \times \widehat{\Delta}_{K}^{k} / K}^{p, q}:=$ $\operatorname{pr}_{X}^{*} \Omega_{X / K}^{p} \otimes \operatorname{pr}_{\widehat{\Delta}_{K}^{k}}^{*} \Omega_{\widehat{\Delta}_{K}^{k} / K}^{q}$, and hence the filtration $F_{X}^{\bullet} \Omega^{*}\left(X \times \widehat{\Delta}_{K}^{k}\right)$ with respect to the first degree. This induces a filtration $F^{\bullet}$ on $D^{*}\left(Y_{\bullet}\right)$, and $I$ is a filtered quasiisomorphism when $\Omega^{*}\left(Y_{\bullet}\right)$ carries the filtration given by $F^{n} \Omega^{*}\left(Y_{\bullet}\right)=\Omega^{\geqslant n}\left(Y_{\bullet}\right)$.

7.6. Let $X$ be an affine scheme in $\mathrm{Sm}_{R}$ with generic fibre $X_{K}$. Recall the complexes $F^{n} R \Gamma_{\mathrm{dR}}\left(X_{K} / K\right)$ from (4.6). Similarly as in (4.5), (4.7), there is a natural map

$$
F^{n} R \Gamma_{\mathrm{dR}}\left(X_{K} / K\right) \rightarrow \Gamma\left(X_{K}, \operatorname{Gd}_{P t\left(X_{K}^{\dagger}\right) \sqcup P t\left(X_{K}\right)} \Omega_{X_{K} / K}^{\geqslant n}\right),
$$

where the complex on the right computes the hypercohomology of $\Omega_{X_{K} / K}^{\geqslant n}$ on $X_{K}$. Since $X_{K}$ is affine, the sheaves $\Omega_{X_{K} / K}^{i}$ are acyclic, and hence the coaugmentation

$$
\Gamma\left(X_{K}, \operatorname{Gd}_{P t\left(X_{K}^{\dagger}\right) \cup P t\left(X_{K}\right)} \Omega_{X_{K} / K}^{\geqslant n}\right) \leftarrow \Gamma\left(X_{K}, \Omega_{X_{K} / K}^{\geqslant n}\right)
$$

is a quasi-isomorphism. The maps (7.7), (7.8), and

$$
\Gamma\left(X_{K}, \Omega_{X_{K} / K}^{\geqslant n}\right) \rightarrow \Gamma\left(X_{K}^{\dagger}, \Omega_{X_{K}^{\dagger} / K}^{\geqslant n}\right) \rightarrow \Gamma\left(\widehat{X}_{K}, \Omega_{\widehat{X}_{K} / K}^{\geqslant n}\right)
$$

give a chain of morphisms connecting $F^{n} R \Gamma_{\mathrm{dR}}\left(X_{K} / K\right)$ with $\Gamma\left(\widehat{X}_{K}, \Omega_{\widehat{X}_{K} / K}^{\geqslant n}\right)$, where the arrow (7.8) pointing in the wrong direction is a quasi-isomorphism. All these morphisms are functorial in $X \in \mathrm{Sm}_{R}$. We denote this by

$$
F^{n} R \Gamma_{\mathrm{dR}}\left(X_{K} / K\right) \rightarrow \stackrel{\simeq}{\leftarrow} \Gamma\left(\widehat{X}_{K}, \Omega_{\widehat{X}_{K} / K}^{\geqslant n}\right) .
$$

If $Y_{\bullet}$ is a simplicial smooth affine $R$-scheme, this and the quasi-isomorphism $I$ from (7.5) give a natural chain of morphisms

$$
F^{n} R \Gamma_{\mathrm{dR}}\left(Y_{K, \bullet} / K\right) \rightarrow \stackrel{\simeq}{\leftarrow} F^{n} D^{*}\left(\widehat{Y}_{K, \bullet}\right) .
$$


We apply (7.10) to $B . \mathrm{GL}_{r, R}$ to get a map

$$
F^{n} H_{\mathrm{dR}}^{2 n}\left(B_{\bullet} \mathrm{GL}_{r, K} / K\right) \rightarrow H^{2 n}\left(F^{n} D^{*}\left(B \cdot \widehat{\mathrm{GL}}_{r, K}\right)\right) .
$$

Similarly as in Lemma 5.14, there is an isomorphism

$$
H^{2 n}\left(\operatorname{mf}\left(F^{n} D^{*}\left(B_{\bullet} \widehat{\mathrm{GL}}_{r, K}\right) \rightarrow D^{*}\left(E_{\bullet} \widehat{\mathrm{GL}}_{r, K}\right)\right)\right) \stackrel{\cong}{\rightarrow} H^{2 n}\left(F^{n} D^{*}\left(B_{\bullet} \widehat{\mathrm{GL}}_{r, K}\right)\right),
$$

and we define

$$
\operatorname{ch}_{n,(r)}^{\mathrm{Kar}} \in H^{2 n}\left(\operatorname{mf}\left(F^{n} D^{*}\left(B \cdot \widehat{\mathrm{GL}}_{r, K}\right) \rightarrow D^{*}\left(E \cdot \widehat{\mathrm{GL}}_{r, K}\right)\right)\right)
$$

to be the unique element whose image under (7.12) coincides with the image of $\mathrm{ch}_{n,(r)}^{\mathrm{dR}}$ under (7.11).

REMARK. Originally, Karoubi used Chern-Weil theory to construct the relevant characteristic classes. This is the reason for the use of the differential graded algebra $D^{*}\left(B . \widehat{G L}_{K}\right)$. One advantage is that it gives more explicit formulae for the relative Chern character. For instance, these are used for the comparison theorem in [Tam12a] and in the work of Choo and Snaith [CS11]. It was checked in [Tam10, Proposition 5.13] that the present approach yields the same classes as the Chern-Weil theoretic one.

To describe Karoubi's version of the relative Chern character we first need a lemma. Let $X=\operatorname{Spec}(A)$ be a regular affine $R$-scheme. We consider $B . \operatorname{GL}(A)$ as a bisimplicial set which is constant in the second direction. Using this, we define the bisimplicial set $F^{b}(A):=B_{\bullet} \operatorname{GL}(A) \times_{B_{\bullet} \mathrm{GL}\left(A^{\dagger}\left\langle\Delta^{\bullet}\right\rangle^{\dagger}\right)} E_{\bullet} \operatorname{GL}\left(A^{\dagger}\left\langle\Delta^{\bullet}\right\rangle^{\dagger}\right)$, and similarly $F_{r}^{\mathrm{b}}(A)$ for every finite level $r$. These are bisimplicial subsets of $F(A)$ (respectively, $F_{r}(A)$ ), from (3.4) and (3.5).

LEMMA 7.14. The induced map on complexes $\mathbf{Z} F^{b}(A) \rightarrow \mathbf{Z F}(A)$ is a quasiisomorphism.

Proof. By the Eilenberg-Zilber theorem it suffices to show that $\mathbf{Z} \operatorname{diag} F^{\mathrm{b}}(A) \rightarrow$ $\mathbf{Z} \operatorname{diag} F(A)$ is a quasi-isomorphism. For this, it is enough to show that the map $\operatorname{diag} F^{b}(A) \rightarrow \operatorname{diag} F(A)$ is acyclic. By the definitions, we have a pullback square

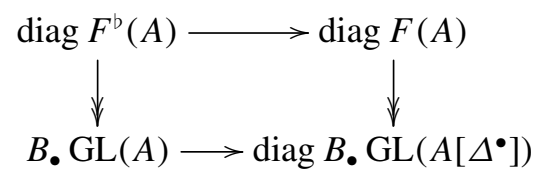


Since diag $E_{\bullet} \operatorname{GL}\left(A^{\dagger}\left\langle\Delta^{\bullet}\right\rangle^{\dagger}\right) \rightarrow \operatorname{diag} B_{\bullet} \operatorname{GL}\left(A^{\dagger}\left\langle\Delta^{\bullet}\right\rangle^{\dagger}\right)$ is a Kan fibration, so are the vertical maps in (7.15). It thus suffices to show that the lower horizontal map is acyclic (see [Ber82, (4.1)]).

Let $(.)^{+}$denote Quillen's plus construction. Then $\pi_{i}\left(B_{\bullet} \operatorname{GL}(A)^{+}\right)=K_{i}(A)$, $i \geqslant 1$, are the Quillen K-groups of $A$. The map $B_{\bullet} \mathrm{GL}(A) \rightarrow B_{\bullet} \mathrm{GL}(A)^{+}$is acyclic. We have a commutative diagram

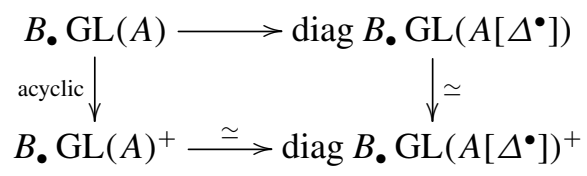

where the lower horizontal map is a weak equivalence by the homotopy invariance for K-theory of regular rings, and the right vertical map is a weak equivalence since $B \cdot \operatorname{GL}\left(A\left[\Delta^{\bullet}\right]\right)$ has the homotopy type of an H-space. Hence the upper horizontal map is acyclic, as desired.

Karoubi's relative Chern character $\mathrm{ch}_{n, i}^{\mathrm{Kar}}$ is defined as the composition

$$
\begin{aligned}
K_{i}^{\text {rel }}(X) & \rightarrow H_{i}(\operatorname{diag} F(A), \mathbf{Z}) \\
& \cong H_{i}\left(\operatorname{diag} F^{\mathrm{b}}(A), \mathbf{Z}\right) \\
& \cong \underset{r}{\lim _{\longrightarrow}} H_{i}\left(\operatorname{diag} F_{r}^{b}(A), \mathbf{Z}\right) \\
& \rightarrow H^{2 n-i-1}\left(\Omega^{<n}\left(\widehat{X}_{K}\right)\right)
\end{aligned}
$$

where the last map is constructed as follows (see [Tam10, Remark 3.6(ii)]): an $i$-simplex $\sigma$ in diag $F_{r}^{b}(A)$ defines a pair of morphisms $\widehat{X}_{K} \stackrel{\sigma_{0}}{\rightarrow} B_{i} \widehat{\mathrm{GL}}_{r, K}$ and $\widehat{X}_{K} \times$ $\widehat{\Delta}_{K}^{i} \stackrel{\sigma_{1}}{\rightarrow} E_{i} \widehat{\mathrm{GL}}_{r, K}$ such that

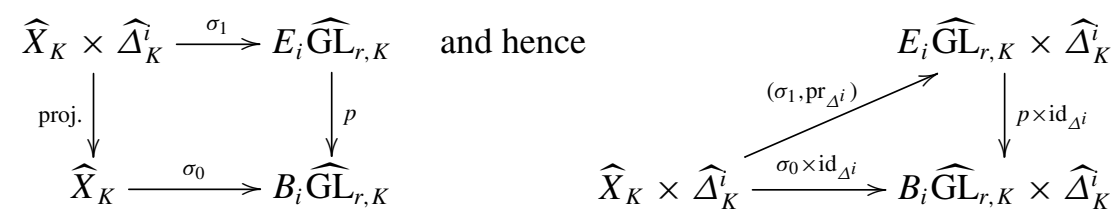

commute. We can write

$$
\operatorname{ch}_{n,(r)}^{\mathrm{Kar}}=\left(\omega_{0}, \omega_{1}\right)
$$

with $\omega_{0} \in F^{n} D^{2 n}\left(B . \widehat{\mathrm{GL}}_{r, K}\right), \omega_{1} \in D^{2 n}\left(E \cdot \widehat{\mathrm{GL}}_{r, K}\right)$ and $d \omega_{1}=p^{*} \omega_{0}$. Then

$$
\begin{aligned}
& \left(\left(\sigma_{0} \times \mathrm{id}_{\Delta^{i}}\right)^{*} \omega_{0},\left(\sigma_{1}, \mathrm{pr}_{\Delta^{i}}\right)^{*} \omega_{1}\right)
\end{aligned}
$$

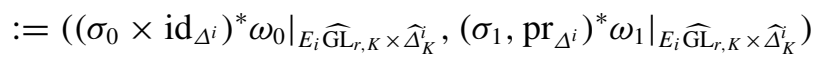

is a cycle of degree $2 n \operatorname{in} \operatorname{mf}\left(F_{\widehat{X}_{K}}^{n} \Omega^{*}\left(\widehat{X}_{K} \times \widehat{\Delta}_{K}^{i}\right) \rightarrow \Omega^{*}\left(\widehat{X}_{K} \times \widehat{\Delta}_{K}^{i}\right)\right)$. We now 
integrate along $\Delta^{i}$ and use the quasi-isomorphism $\operatorname{mf}\left(\Omega^{\geqslant n}\left(\widehat{X}_{K}\right) \rightarrow \Omega^{*}\left(\widehat{X}_{K}\right)\right) \stackrel{\sim}{\rightarrow}$ $\Omega^{<n}\left(\widehat{X}_{K}\right)[-1]$ induced by the projection to the second component to get the map

$$
F_{r}^{\mathrm{b}}(A) \ni \sigma \mapsto \int_{\Delta^{i}}\left(\sigma_{1}, \mathrm{pr}_{\Delta^{i}}\right)^{*} \omega_{1} \in \Omega^{<n}\left(\widehat{X}_{K}\right)^{2 n-i-1} .
$$

This induces a well-defined homomorphism

$$
H_{i}\left(\operatorname{diag} F_{r}^{b}(A), \mathbf{Z}\right) \rightarrow H^{2 n-i-1}\left(\Omega^{<n}\left(\widehat{X}_{K}\right)\right)
$$

compatible for varying $r$.

To compare $\mathrm{ch}_{n, i}^{\mathrm{Kar}}$ with our version of the relative Chern character, we need the following.

LEMMA 7.18. Let $X$ be a smooth affine $R$-scheme. There is a natural map

$$
H_{\text {rel }}^{*}(X, n) \rightarrow H^{*}\left(\operatorname{mf}\left(\Omega^{\geqslant n}\left(\widehat{X}_{K}\right) \rightarrow \Omega^{*}\left(\widehat{X}_{K}\right)\right)\right) \cong H^{*-1}\left(\Omega^{<n}\left(\widehat{X}_{K}\right)\right) .
$$

Proof. We have $H_{\text {rel }}^{*}(X, n)=\operatorname{mf}\left(F^{n} R \Gamma_{\mathrm{dR}}\left(X_{K} / K\right) \rightarrow R \Gamma_{\mathrm{dR}}\left(\widehat{X}_{K} / K\right)\right)$. Since $X$ is affine, we have the natural chain of morphisms $F^{n} R \Gamma_{\mathrm{dR}}\left(X_{K} / K\right) \rightarrow \stackrel{\simeq}{\longleftarrow} \Omega^{\geqslant n}\left(\widehat{X}_{K}\right)$ from (7.9) and similarly $R \Gamma_{\mathrm{dR}}\left(\widehat{X}_{K} / K\right) \stackrel{\simeq}{\longleftarrow} \Omega^{*}\left(\widehat{X}_{K}\right)$. These together induce the desired chain of maps,

$$
\operatorname{mf}\left(F^{n} R \Gamma_{\mathrm{dR}}\left(X_{K} / K\right) \rightarrow R \Gamma_{\mathrm{dR}}\left(\widehat{X}_{K} / K\right)\right) \rightarrow \stackrel{\simeq}{\leftarrow} \operatorname{mf}\left(\Omega^{\geqslant n}\left(\widehat{X}_{K}\right) \rightarrow \Omega^{*}\left(\widehat{X}_{K}\right)\right) .
$$

THEOREM 7.20. Let $X$ be a smooth affine R-scheme. The composition of the relative Chern character $\mathrm{ch}_{n, i}^{\mathrm{rel}}$ of Definition 4 with the comparison map (7.19) coincides with Karoubi's relative Chern character $\mathrm{ch}_{n, i}^{\mathrm{Kar}}$.

Proof. Let $X=\operatorname{Spec}(A)$. Using the quasi-isomorphism $\mathbf{Z} F^{b}(A) \stackrel{\simeq}{\rightarrow} \mathbf{Z} F(A)$, we can describe the composition

$$
K_{i}^{\mathrm{rel}}(X) \stackrel{\mathrm{ch}_{n, i}^{\mathrm{rel}}}{\longrightarrow} H_{\mathrm{rel}}^{2 n-i}(X, n) \stackrel{(7.19)}{\longrightarrow} H^{2 n-i-1}\left(\Omega^{<n}\left(\widehat{X}_{K}\right)\right)
$$

explicitly, as follows. We have a natural map (see the construction in Lemma 7.18)

$$
\begin{gathered}
H^{2 n}\left(\operatorname{mf}\left(F^{n} R \Gamma_{\mathrm{dR}}\left(B \cdot \mathrm{GL}_{r, K} / K\right) \rightarrow R \Gamma_{\mathrm{dR}}\left(E_{\bullet} \widehat{\mathrm{GL}}_{r, K}\right)\right)\right) \\
\quad \rightarrow H^{2 n}\left(\operatorname{mf}\left(\Omega^{\geqslant n}\left(B \cdot \widehat{\mathrm{GL}}_{r, K}\right) \rightarrow \Omega^{*}\left(E_{\bullet} \widehat{\mathrm{GL}}_{r, K}\right)\right)\right),
\end{gathered}
$$

and we denote the image of $\operatorname{ch}_{n,(r)}^{\text {rel }}($ see (5.15)) under this map by

$$
\widetilde{c h}_{n,(r)}^{\text {rel }} \in H^{2 n}\left(\operatorname{mf}\left(\Omega \geqslant n\left(B . \widehat{G L}_{r, K}\right) \rightarrow \Omega^{*}\left(E . \widehat{G L}_{r, K}\right)\right)\right) \text {. }
$$


As in (5.7), we have a map

$$
H_{i}\left(\operatorname{Tot} \mathbf{Z} F_{r}^{b}(A)\right) \rightarrow \operatorname{Hom}\left(\begin{array}{c}
H^{2 n}\left(\operatorname{mf}\left(\Omega \geqslant n\left(B . \widehat{\mathrm{GL}}_{r, K}\right) \rightarrow \Omega^{*}\left(E_{\bullet} \widehat{\mathrm{GL}}_{r, K}\right)\right)\right), \\
H^{2 n-i}\left(\operatorname{mf}\left(\Omega \geqslant n\left(\widehat{X}_{K}\right) \rightarrow \Omega^{*}\left(\widehat{X}_{K} \times \widehat{\Delta}_{K}^{\bullet}\right)\right)\right)
\end{array}\right),
$$

which we can compose with the evaluation eval ${\widetilde{\mathrm{ch}_{n,(r)}^{\text {rel }}}}$ to get

$$
H_{i}\left(\operatorname{Tot} \mathbf{Z} F_{r}^{\mathrm{b}}(A)\right) \rightarrow H^{2 n-i}\left(\operatorname{mf}\left(\Omega^{\geqslant n}\left(\widehat{X}_{K}\right) \rightarrow \Omega^{*}\left(\widehat{X}_{K} \times \widehat{\Delta}_{K}^{\bullet}\right)\right)\right) .
$$

Since $\widehat{X}_{K} \times \widehat{\Delta}_{K}^{p}$ is an affinoid dagger space, and hence the higher cohomology of coherent sheaves on $\widehat{X}_{K} \times \widehat{\Delta}_{K}^{p}$ vanishes, the argument of Lemma 5.16 shows that the coaugmentation $\Omega^{*}\left(\widehat{X}_{K}\right) \rightarrow \Omega^{*}\left(\widehat{X}_{K} \times \widehat{\Delta}_{K}^{\bullet}\right)$ is a quasi-isomorphism. It induces an isomorphism

$$
H^{2 n-i}\left(\operatorname{mf}\left(\Omega^{\geqslant n}\left(\widehat{X}_{K}\right) \rightarrow \Omega^{*}\left(\widehat{X}_{K} \times \widehat{\Delta}_{K}^{\bullet}\right)\right)\right) \cong H^{2 n-i-1}\left(\Omega^{<n}\left(\widehat{X}_{K}\right)\right) .
$$

Now (7.21) equals the composition of

$$
\begin{aligned}
K_{i}^{\text {rel }}(X) & =\pi_{i}(F(A)) \stackrel{\text { Hurewicz }}{\longrightarrow} H_{i}(\mathbf{Z} \operatorname{diag} F(A)) \\
& \stackrel{7.14}{\cong} H_{i}\left(\mathbf{Z} \operatorname{diag} F^{\mathrm{b}}(A)\right) \stackrel{\text { Eilenberg-Zilber }}{\cong} H_{i}\left(\operatorname{Tot} \mathbf{Z} F^{\mathrm{b}}(A)\right) \\
& \cong \underset{r}{\stackrel{\lim }{\longrightarrow}} H_{i}\left(\operatorname{Tot} \mathbf{Z} F_{r}^{\mathrm{b}}(A)\right)
\end{aligned}
$$

with the map induced on the direct limit by (7.23) and (7.24).

From the definitions of $\operatorname{ch}_{n,(r)}^{\text {rel }}$ in (5.15) and $\operatorname{ch}_{n,(r)}^{\mathrm{Kar}}$ in (7.13), it is clear that $\widetilde{c h}_{n,(r)}^{\mathrm{rel}}=I\left(\mathrm{ch}_{n,(r)}^{\mathrm{Kar}}\right)$, where we still denote by $I$ the isomorphism induced by $I$ (see (7.5)) on the cohomology of the respective mapping fibres.

Thus we have to show that

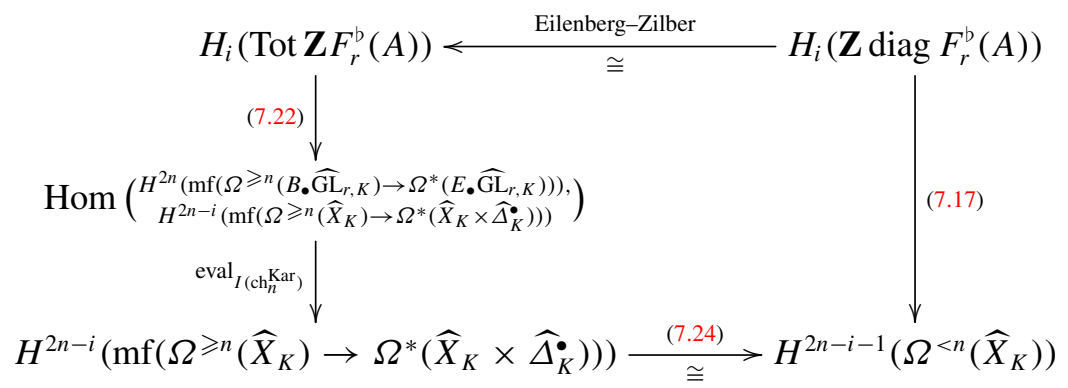

commutes. The following lemma shows that, on the level of complexes, (7.24) is given by sending an element of the mapping fibre $\left(\eta_{0}, \eta_{1}\right)$ to the integral of $\eta_{1}$ along the simplex $\Delta^{\bullet}$. 
LEMMA 7.27. A quasi-inverse of $\Omega^{*}\left(\widehat{X}_{K}\right) \stackrel{\simeq}{\rightarrow} \Omega^{*}\left(\widehat{X}_{K} \times \widehat{\Delta}_{K}^{\bullet}\right)$ is given by integration over the standard simplices:

$$
\Omega^{k+p}\left(\widehat{X}_{K} \times \widehat{\Delta}_{K}^{p}\right) \rightarrow \Omega^{k}\left(\widehat{X}_{K}\right), \quad \omega \mapsto(-1)^{p(p-1) / 2} \int_{\Delta^{p}} \omega
$$

Proof. The integration map is obviously left inverse to the inclusion of $\Omega^{*}\left(\widehat{X}_{K}\right)$ as the zeroth column in the double complex $\Omega^{*}\left(\widehat{X}_{K} \times \widehat{\Delta}_{K}^{\bullet}\right)$. So we only have to check that the integration indeed defines a morphism of complexes. This is straightforward using the following relative version of Stokes' formula,

$$
\int_{\Delta^{p}} d \omega=(-1)^{p} d \int_{\Delta^{p}} \omega+\sum_{i=0}^{p}(-1)^{i} \int_{\Delta^{p-1}}\left(\partial^{i}\right)^{*} \omega,
$$

and keeping in mind that the total differential on $\Omega^{q}\left(\widehat{X}_{K} \times \widehat{\Delta}_{K}^{p}\right)$ is given by $\omega \mapsto$ $(-1)^{p} d \omega+\sum_{i=0}^{p}(-1)^{i}\left(\partial^{i}\right)^{*} \omega$.

To show the commutativity of diagram (7.26), we start with a bisimplex $\sigma \in F_{r}^{b}(A)_{p, i-p}$, so the degree of $\sigma$ in $\operatorname{Tot} \mathbf{Z} F_{r}^{b}(A)$ is $i$. First, we compute its image in $\Omega^{<n}\left(\widehat{X}_{K}\right)$ going counterclockwise. As before, $\sigma$ gives a commutative diagram

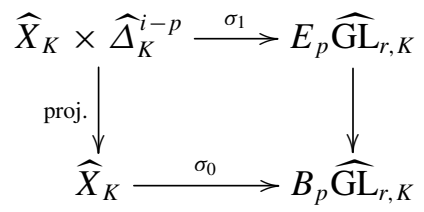

The component of $I\left(\mathrm{ch}_{n,(r)}^{\mathrm{Kar}}\right)$ in simplicial degree $p$ is given by $\left(\int_{\Delta^{p}} \omega_{0}, \int_{\Delta^{p}} \omega_{1}\right)$ (see (7.16)). Hence the image of $\sigma$ in the lower left corner of (7.26) is given by $(-1)^{(i-p)(i-p-1) / 2}\left(\sigma_{0}^{*} \int_{\Delta^{p}} \omega_{0}, \sigma_{1}^{*} \int_{\Delta^{p}} \omega_{1}\right) \in \operatorname{mf}\left(\Omega^{\geqslant n}\left(\widehat{X}_{K}\right) \rightarrow \Omega^{*}\left(\widehat{X}_{K} \times \widehat{\Delta}_{K}^{i-p}\right)\right)^{2 n-p}$, and its image in $\Omega^{<n}\left(\widehat{X}_{K}\right)$ by

$$
\int_{\Delta^{i-p}} \sigma_{1}^{*} \int_{\Delta^{p}} \omega_{1}=\int_{\Delta^{i-p} \times \Delta^{p}}\left(\sigma_{1} \times \mathrm{id}_{\Delta^{p}}\right)^{*} \omega_{1} .
$$

Note that there is no sign, because the signs introduced via (7.22) (see (5.6)) and the integration map of Lemma 7.27 cancel out.

Next, we compute the image of $\sigma$ going through diagram (7.26) clockwise. To do this, we use the shuffle map which is an inverse of the Eilenberg-Zilber isomorphism (see [Wei94, 8.5.4]): recall that a $(p, i-p)$ shuffle $(\mu, v)$ is a permutation $\left(\mu_{1}, \mu_{2}, \ldots, \mu_{p}, v_{1}, \ldots, v_{i-p}\right)$ of $\{1,2, \ldots, i\}$ such that $\mu_{1}<\mu_{2}<$ $\cdots<\mu_{p}$ and $v_{1}<\cdots<v_{i-p}$. It determines a map $\mu:[i] \rightarrow[p]$ in the category 
$\Delta$ given by $\mu=s^{\mu_{1}-1} \circ s^{\mu_{2}-1} \circ \cdots \circ s^{\mu_{p}-1}$ and similarly $v:[i] \rightarrow[i-p]$ such that $(\mu, v):[i] \rightarrow[p] \times[i-p]$ is a nondegenerate $i$-simplex of the simplicial set $\Delta^{p} \times \Delta^{i-p}$. All such simplices arise in this way. On any simplicial (respectively, cosimplicial) object, we have induced maps $\mu^{*}, \nu^{*}$ (respectively, $\mu_{*}, v_{*}$ ).

In particular, we have $\mu^{*}: E_{p} \widehat{\mathrm{GL}}_{r, K} \rightarrow E_{i} \widehat{\mathrm{GL}}_{r, K}, \mu^{*}: B_{p} \widehat{\mathrm{GL}}_{r, K} \rightarrow B_{i} \widehat{\mathrm{GL}}_{r, K}$, and $\nu_{*}: \widehat{\Delta}_{K}^{i} \rightarrow \widehat{\Delta}_{K}^{i-p}$. Recall that we started with a $(p, i-p)$-simplex $\sigma$ in $F_{r}^{b}(A)$. Then $(\mu, v)^{*} \sigma$ is an $i$-simplex in $\operatorname{diag} F_{r}^{b}(A)$. The shuffle map sends $\sigma$ to

$$
\sum_{(\mu, v)} \operatorname{sgn}(\mu, v)(\mu, v)^{*} \sigma
$$

where the sum runs over all $(p, i-p)$-shuffles $(\mu, v)$. Its image under the Karoubi construction $(7.17)$ is then given by

$$
\left.\sum_{(\mu, v)} \operatorname{sgn}(\mu, \nu) \int_{\Delta^{i}}\left(\left((\mu, v)^{*} \sigma\right)_{1}, \mathrm{pr}_{\Delta^{i}}\right)^{*} \omega_{1}\right|_{E_{i} \widehat{\mathrm{GL}}_{r, K} \times \widehat{\Delta}_{K}^{i}} .
$$

Thus, to complete the proof, we have to show the equality

$$
\begin{aligned}
& \left.\int_{\Delta^{i-p} \times \Delta^{p}}\left(\sigma_{1} \times \mathrm{id}_{\Delta^{p}}\right)^{*} \omega_{1}\right|_{E_{p} \widehat{\mathrm{GL}}_{K} \times \widehat{\Delta}_{K}^{p}} \\
& =\left.\sum_{(\mu, \nu)} \operatorname{sgn}(\mu, v) \int_{\Delta^{i}}\left(\left((\mu, \nu)^{*} \sigma\right)_{1}, \mathrm{pr}_{\Delta^{i}}\right)^{*} \omega_{1}\right|_{E_{i} \widehat{\mathrm{GL}}_{K} \times \widehat{\Delta}_{K}^{i}}
\end{aligned}
$$

Describing the map that appears in the right-hand side of the formula more explicitly, we have that for a $(p, i-p)$-shuffle $(\mu, v)$ the induced map $\widehat{X}_{K} \times$ $\widehat{\Delta}_{K}^{i} \stackrel{\left((\mu, \nu)^{*} \sigma\right)_{1}}{\longrightarrow} E_{i} \widehat{\mathrm{GL}}_{K}$ is given by $\mu^{*} \circ \sigma_{1} \circ\left(\operatorname{id}_{\widehat{X}_{K}} \times v_{*}\right)$. Hence we have a commutative diagram,

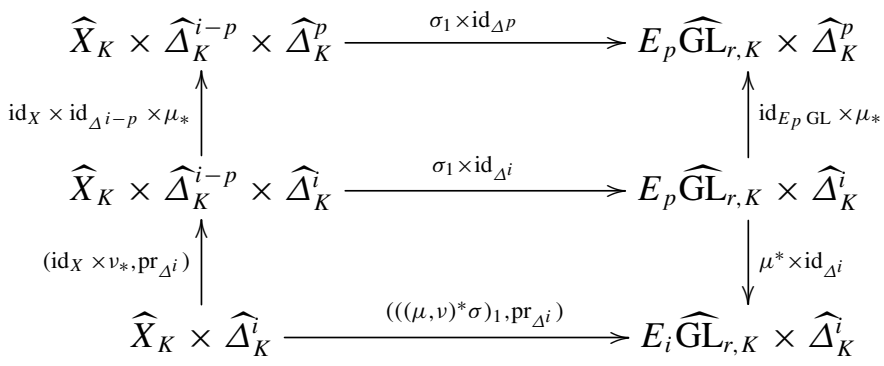

The composition of the two left vertical arrows is $\operatorname{id}_{\widehat{X}_{K}} \times\left(v_{*}, \mu_{*}\right)$. The map $\left(v_{*}, \mu_{*}\right)$ is exactly the map corresponding to the shuffle $(\mu, v)$ in the standard 
decomposition of $\Delta^{i-p} \times \Delta^{p}$ into $i$-simplices. It follows (see (7.3)) that

$$
\int_{\Delta^{i-p} \times \Delta^{p}}(?)=\sum_{(\mu, v)} \operatorname{sgn}(\mu, \nu) \int_{\Delta^{i}}\left(\operatorname{id}_{X} \times\left(v_{*}, \mu_{*}\right)\right)^{*}(?) .
$$

Since $\omega_{1} \in D^{2 n-1}\left(E . \widehat{\mathrm{GL}}_{r, K}\right)$ is a simplicial differential form, we have ( $\mu^{*} \times$

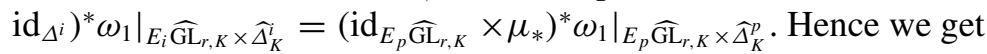

$$
\left.\left(\operatorname{id}_{X} \times\left(v_{*}, \mu_{*}\right)\right)^{*}\left(\sigma_{1} \times \mathrm{id}_{\Delta^{p}}\right)^{*} \omega_{1}\right|_{E_{p} \widehat{\mathrm{GL}}_{r, K} \times \Delta^{p}}=\left.\left(\left((\mu, \nu)^{*} \sigma\right)_{1}, \mathrm{pr}_{\Delta^{i}}\right)^{*} \omega_{1}\right|_{E_{i} \widehat{\mathrm{GL}}_{r, K} \times \Delta^{i}} .
$$

Setting (?) $=\left.\left(\sigma_{1} \times \mathrm{id}_{\Delta^{p}}\right)^{*} \omega_{1}\right|_{E_{p} \widehat{\mathrm{GL}}_{r, K} \times \Delta^{p}}$ in (7.29), we get the desired equality (7.28).

\section{Acknowledgements}

The results presented here emerged from my thesis [Tam10]. It is a pleasure to thank my advisor Guido Kings for his interest and constant support. I would like to thank the California Institute of Technology, where most of this work was done, and especially Matthias Flach, for their hospitality, and the Deutsche Forschungsgemeinschaft for financial support. Furthermore, I would like to thank Nicola Mazzari for interesting discussions about this work, and the referee for several useful remarks.

\section{References}

[Ayo] J. Ayoub, Motifs des variétés analytiques rigides, preprint available at http://user.math.uzh.ch/ayoub/.

[BdJ12] A. Besser and R. de Jeu, 'The syntomic regulator for $K_{4}$ of curves', Pacific J. Math. 260 (2) (2012), 305-380.

[Be154] A. Berlinson, 'Higher regulators and values of L-functions', in Current Problems in Mathematics, Vol. 24 (Itogi Nauki i Tekhniki, Akad Nauk SSSR Vsesoyuz. Inst. Nauchn. i Tekhn. Inform., Moscow, 1984), 181-238.

[Ber82] A. Jon Berrick, An Approach to Algebraic K-Theory, Research Notes in Mathematics, 56 (Pitman (Advanced Publishing Program), Boston, MA, 1982).

[Ber96] P. Berthelot, Cohomologie rigide et cohomologie rigide à supports propres, Première partie (version provisoire 1991), Prépublication IRMAR 96-03, January 1996.

[Ber97] P. Berthelot, 'Finitude et pureté cohomologique en cohomologie rigide', Invent. Math. 128 (2) (1997), 329-377. With an appendix in English by Aise Johan de Jong.

[Bes00a] A. Besser, 'A generalization of Coleman's p-adic integration theory', Invent. Math. 142 (2) (2000), 397-434.

[Bes00b] A. Besser, 'Syntomic regulators and $p$-adic integration. I. Rigid syntomic regulators', Israel J. Math. 120 (part B) (2000), 291-334.

[Bes12] A. Besser, 'On the syntomic regulator for $K_{1}$ of a surface', Israel J. Math. 190 (2012), 29-66. 
[Bor74] A. Borel, 'Stable real cohomology of arithmetic groups', Ann. Sci. Éc. Norm. Supér. (4) 7 (1974), 235-272. (1975).

[Cal85] A. Calvo, ' $K$-théorie des anneaux ultramétriques', C. R. Acad. Sci. Paris Sér. I Math. 300 (14) (1985), 459-462.

[CCM13] B. Chiarellotto, A. Ciccioni and N. Mazzari, 'Cycle classes and the syntomic regulator', Algebra Number Theory 7 (3) (2013), 533-566.

[CK88] A. Connes and M. Karoubi, 'Caractère multiplicatif d'un module de Fredholm', K-Theory 2 (3) (1988), 431-463.

[CLS98] B. Chiarellotto and B. Le Stum, 'Sur la pureté de la cohomologie cristalline', C. $R$. Acad. Sci. Paris Sér. I Math. 326 (8) (1998), 961-963.

[CS11] Z. Choo and V. Snaith, ' $p$-adic cocycles and their regulator maps', J. K-Theory 8 (2) (2011), 241-249.

[DM12] F. Déglise and N. Mazzari, The rigid syntomic ring spectrum, J. Inst. Math. Jussieu, available on CJO2014. doi:10.1017/S1474748014000152.

[Del71] P. Deligne, 'Théorie de Hodge, II', Inst. Hautes Études Sci. Publ. Math. 40 (1971), 5-57.

[Fal89] G. Faltings, 'Crystalline cohomology and p-adic Galois-representations', in Algebraic Analysis, Geometry, and Number Theory (Baltimore, MD, 1988) (John Hopkins University Press, Baltimore, MD, 1989), 25-80.

[Ger73] S. M. Gersten, 'Higher $K$-theory of rings', in Algebraic K-Theory, I: Higher K-Theories (Proc. Conf. Seattle Res. Center, Battelle Memorial Inst., 1972), Lecture Notes in Mathematics, 341 (Springer, Berlin, 1973), 3-42.

[GK99] E. Große-Klönne, de Rham-Komologie in der rigiden Analysis, Preprintreihe des SFB 478 - Geometrische Strukturen in der Mathematik, Münster, Heft 39, 1999.

[GK00] E. Große-Klönne, 'Rigid analytic spaces with overconvergent structure sheaf', J. Reine Angew. Math. 519 (2000), 73-95.

[Gro94] M. Gros, 'Régulateurs syntomiques et valeurs de fonctions $L$ p-adiques II', Invent. Math. 115 (1) (1994), 61-79.

[Ham00] N. Hamida, 'Description explicite du régulateur de Borel', C. R. Acad. Sci. Paris Sér. I Math. 330 (3) (2000), 169-172.

[Ham06] N. Hamida, 'Le régulateur p-adique', C. R. Math. Acad. Sci. Paris 342 (11) (2006), 807-812.

[Har77] G. Harder, 'Die Kohomologie $S$-arithmetischer Gruppen über Funktionenkörpern', Invent. Math. 42 (1977), 135-175.

[HK11] A. Huber and G. Kings, 'A p-adic analogue of the Borel regulator and the Bloch-Kato exponential map', J. Inst. Math. Jussieu 10 (1) (2011), 149-190.

[Hub95] A. Huber, 'Mixed motives and their realization in derived categories', in Lecture Notes in Mathematics, 1604 (Springer, Berlin, 1995).

[Ivo05] F. Ivorra, Réalisations $\ell$-adique des motifs mixtes, PhD Thesis, Université Paris VI Pierre et Marie Curie, 2005.

[Kar82] M. Karoubi, 'Connexions, courbures et classes caractéristiques en $K$-théorie algébrique', in Current Trends in Algebraic Topology, Part 1, London, Ont., 1981, CMS Conf. Proc., 2 (American Mathematical Society, Providence, RI, 1982), 19-27.

[Kar83] M. Karoubi, 'Homologie cyclique et régulateurs en $K$-théorie algébrique', C. R. Acad. Sci. Paris Sér. I Math. 297 (10) (1983), 557-560.

[Kar87] M. Karoubi, 'Homologie cyclique et $K$-théorie', Astérisque (149) (1987), 1-147.

[KV71] M. Karoubi and O. Villamayor, ' $K$-théorie algébrique et $K$-théorie topologique I', Math. Scand. 28 (1971), 265-307. (1972). 
[Mer72] D. Meredith, 'Weak formal schemes', Nagoya Math. J. 45 (1972), 1-38.

[MW68] P. Monsky and G. Washnitzer, 'Formal cohomology I', Ann. of Math. (2) 88 (1968), 181-217.

[Niz97] W. Niziol, 'On the image of p-adic regulators', Invent. Math. 127 (2) (1997), 375-400.

[Niz01] W. Nizioł, 'Cohomology of crystalline smooth sheaves', Compos. Math. 129 (2) (2001), 123-147.

[Qui72] D. Quillen, 'On the cohomology and $K$-theory of the general linear groups over a finite field', Ann. of Math. (2) 96 (1972), 552-586.

[Tam10] G. Tamme, The relative Chern character and regulators, $\mathrm{PhD}$ Thesis, Universität Regensburg, 2010, http://epub.uni-regensburg.de/15595/.

[Tam12a] G. Tamme, 'Comparison of Karoubi's regulator and the p-adic Borel regulator', J. K-Theory 9 (03) (2012), 579-600.

[Tam12b] G. Tamme, 'Karoubi's relative Chern character and Beilinson's regulator', Ann. Sci. Éc. Norm. Supér. (4) 45 (4) (2012), 601-636.

[Tho85] R. W. Thomason, 'Algebraic $K$-theory and étale cohomology', Ann. Sci. Éc. Norm. Supér. (4) 18 (3) (1985), 437-552.

[vdPS95] M. van der Put and P. Schneider, 'Points and topologies in rigid geometry', Math. Ann. 302 (1) (1995), 81-103.

[Wei89] C. Weibel, 'Homotopy algebraic K-theory', in Algebraic K-Theory and Algebraic Number Theory (Honolulu, HI, 1987), Contemporary Mathematics, 83 (American Mathematical Society, Providence, RI, 1989), 461-488.

[Wei94] C. Weibel, An Introduction to Homological Algebra, Cambridge Studies in Advanced Mathematics, 38 (Cambridge University Press, Cambridge, 1994).

[Wei97] C. Weibel, 'The Hodge filtration and cyclic homology', K-Theory 12 (2) (1997), 145-164. 